\title{
Differential Tractography as a Track-Based Biomarker for Neuronal Injury
}

2 Authors: Fang-Cheng Yeh ${ }^{1,2, *}$, Islam M. Zaydan ${ }^{3}$, Valerie R. Suski ${ }^{3}$, David Lacomis ${ }^{3,4}$, R. Mark

3 Richardson ${ }^{1}$, Joseph C. Maroon ${ }^{1}$, and Jessica Barrios-Martinez ${ }^{1}$

4 Affiliations:

$5 \quad$ 'Department of Neurological Surgery, University of Pittsburgh School of Medicine, Pittsburgh,

6 Pennsylvania, United States

$7 \quad$ Department of Bioengineering, University of Pittsburgh, Pittsburgh, Pennsylvania, United States

$8{ }^{3}$ Department of Neurology, and the ${ }^{4}$ Live Like Lou Center for ALS Research, University of Pittsburgh

9 School of Medicine, Pittsburgh, PA, United States

$10{ }^{*}$ Correspondence to:

11 Fang-Cheng Yeh, M.D. Ph.D.

12 Department of Neurological Surgery,

13 Department of Bioengineering,

14 University of Pittsburgh, Pittsburgh, Pennsylvania

15 Email: frank.yeh@pitt.edu

16

17 One Sentence Summary:

18 Differential tractography utilizes repeat diffusion MRI scans to identify the exact segment of tracks with a 19 neuronal injury. 


\section{Abstract}

22 Diffusion MRI tractography has been used to map the axonal structure of human brain, but its ability to

23 detect neuronal injury is yet to be explored. Here we report differential tractography, a new type of

24 tractography that utilizes repeat MRI scans and a novel tracking strategy to map the exact segment of 25 fiber pathways with a neuronal injury. We examined differential tractography on multiple sclerosis,

26 Huntington disease, amyotrophic lateral sclerosis, and epileptic patients. The results showed that the

27 affected pathways shown by differential tractography matched well with the unique clinical symptoms of

28 the patients, and the false discovery rate of the findings could be estimated using a sham setting to

29 provide a reliability measurement. This novel approach enables a quantitative and objective method to 30 monitor neuronal injury in individuals, allowing for diagnostic and prognostic evaluation of brain 31 diseases.

Keywords: diffusion MRI, differential tractography, fiber tracking, imaging biomarker, neuronal injury,

34 multiple sclerosis, Huntington's disease, amyotrophic lateral sclerosis, epilepsy. 


\section{Introduction}

Magnetic resonance imaging $(\mathrm{MRI})$ is a commonly used neuroimaging technique for revealing a structural change in patients with neurological disorders. Studies have used structural MRI to reveal gray matter atrophy in patients with multiple sclerosis (Rovira et al., 2015; Wattjes et al., 2015) and atrophy in the caudate in patients with Huntington disease (Tabrizi et al., 2009; Tabrizi et al., 2012). In addition to structural MRI, diffusion MRI has also been explored as an imaging biomarker for early-stage neuronal injury before atrophy happens. Animal studies have used diffusion tensor imaging (DTI)(Basser et al., 1994) to detect acute demyelination or axonal loss (Song et al., 2002; Song et al., 2005). The decrease of anisotropic diffusion has been shown to be correlated with axonal loss (Budde et al., 2009; Huisman et al., 2004; Werring et al., 1999; Werring et al., 2000). However, anisotropy remains a voxel-based measurement, which is prone to local variations such as partial volume effect (Henf et al., 2018; Wang et al., 2011) or signal noise, thereby limiting its clinical applications (Melonakos et al., 2011). Greater specificity and sensitivity could be achieved by aggregating voxel-wise anisotropy changes along fiber pathways and grouping them into a "track".

To this end, we propose a novel method called "differential tractography" to provide a track-based biomarker for neuronal injury. This method compares repeat scans of the same individuals to capture neuronal injury reflected by a decrease of anisotropic diffusion, or "anisotropy" (Fig 1a-1c). To achieve a higher specificity, we imbued the deterministic fiber tracking algorithm (Yeh et al., 2013) with a novel "tracking-the-difference" paradigm. This was realized by adding an additional criterion to track along trajectories on which a decrease of anisotropy was found between repeat scans (Fig. 1d-1e). Integrating this "tracking-the-difference" paradigm into the fiber tracking process resulted in a new tractography 
modality that tracks the exact portion of pathways exhibiting substantial differences in anisotropy. The additional criterion ignored unaffected regions and enhanced meaningful findings related to neuronal injury. In comparison, the conventional fiber tracking (Wedeen et al., 2012) is based on a "tracking-the-existence" paradigm. It only considers anisotropy from one MRI scan and thus will include all existing pathways regardless of whether they have an injury.

To implement differential tractography, the algorithm requires one anisotropy value for each fiber population to calculate its longitudinal change, but the fractional anisotropy (FA) derived from DTI is a voxel-based measurement, and thus all fiber orientations within the same voxel will inherit the same anisotropy value. To overcome this limitation, we used the anisotropic component of the spin distribution function (SDF)(Yeh et al., 2010) as an anisotropy measurement for each fiber population. SDF could provide one anisotropy measurement for each fiber population. This approach has been shown to be more robust against partial volume effect (Yeh et al., 2013) and achieved high accuracy in a recent competition study (ID\#3)(Maier-Hein et al., 2017).

To further maximize the detection power, we used a diffusion MRI acquisition that sampled 22 diffusion sensitizations (b-values) at 257 directions, a substantial improvement over one sensitization at 30 60 directions used in the conventional settings, or 3 diffusion sensitizations at 180 directions used in the current mainstream studies (Glasser et al., 2016). The higher number of diffusion sensitizations greatly

74 increased the chance to detect neuronal injury that involves only a subtle change in the restricted

75 diffusion (Wang et al., 2011). We also introduce a novel sham setting that can estimate the false 76 discovery rate (FDR) of differential tractography to provide a reliability measurement against local 77 random error. 
79 To evaluate the performance, we applied differential tractography to patients with four different clinical

80 scenarios at different stages of neuronal injury (demographic information listed in Table 1). The first

81 scenario was multiple sclerosis (MS) with the first episode of optic neuritis. The baseline scans were

82 acquired right after the onset of the visual symptom, and the follow-up diffusion MRI scans were

83 acquired 6 months after. This scenario tested differential tractography at the early stage of neuronal

84 injury to explore its sensitivity, and any meaningful findings should be located near the visual pathways.

85 The second scenario was the manifested Huntington disease (HD) with worsening clinical motor scores

86 during the interval of their repeat MRI scans. We examined whether differential tractography could

87 detect progressing neuronal injury at striatal pathways that are commonly affected by the disease. The

88 third scenario studied the neuronal injury in an amyotrophic lateral sclerosis (ALS) patient with a

89 deteriorating functional motor score. We examined whether differential tractography could be correlated

90 with the patient's clinical presentation. Last, we applied differential tractography to an epileptic patient

91 treated by anterior temporal lobectomy. The baseline scan was acquired before the surgery, and the

92 follow-up scan was acquired one year after the surgery. This examined whether differential tractography

93 could correctly locate pathways with established neuronal injury after surgery, and meaningful findings

94 should be in pathways previously connected to the area of resection. We also applied differential

95 tractography to a normal subject to demonstrate how differential tractography may capture false results.

96 Material and Methods

97 MRI experiments on clinical patients with neurological disorders

98 The diffusion MRI acquisition included a baseline scan and another follow-up scan (acquired months

99 later) of the same subject. We acquired scans on 6 patients with different neurological diseases 
100 including MS, HD, ALS, and epilepsy, in addition to one healthy volunteer. Table 1 summarizes their

101 basic demographic and scan interval information, and brief medical history of these patients are 102 summarized in Supplementary Materials. The ALS patient was previously reported (Abhinav et al., 103 2014). The diffusion data were acquired on a 3T Tim Trio System (Siemens, Erlangen, Germany) using 104 a pulsed-gradient spin-echo 2D echo-planar imaging sequence. A 32-channel coil was used with a head 105 stabilizer to limit head motion. Each diffusion MRI scan acquired 22 b-values ranging from 0 to 7,000 $106 \mathrm{~s} / \mathrm{mm}^{2}$ at a total of 257 diffusion sampling direction using a q-space imaging scheme (Callaghan, 1991). 107 The in-plane resolution and slice thickness were $2.4 \mathrm{~mm}$. TE=154 $\mathrm{ms}$, and TR=9500 ms. The total 108 scanning time was 45 minutes.

109 This is a retrospective study, and the multi-band sequence was not available previously. Currently, the 110 same protocol using multi-band sequence has a much shorter scanning time of 12 minutes (see 111 Discussion section for details).

\section{Quality control of diffusion MRI data}

113 We applied a series of quality control to minimize possible false results due to acquisition issues. The

114 first quality control was done by checking whether the image dimension, resolution, and b-table were 115 consistent between repeat scans. All scan data were confirmed to have a consistent setting between 116 repeat scans.

117 The second quality control was done by calculating the mean Pearson correlation coefficient of the 118 "neighboring" diffusion-weighted images:

$119 \frac{1}{n} \sum_{i=1}^{n} \rho\left(S_{i}, S_{N(i)}\right)$ 
where $\rho$ calculates the Pearson correlation coefficient, $S_{i}$ is the $i$-th diffusion weighted image, and $N(i)$ 121 returns the index of neigthe hboring diffusion-weighted image acquired by the most similar diffusion 122 sensitization in the q-space:

$$
N(i)=\arg \min _{j, j \neq i}\|\sqrt{b(i)} \vec{g}(i)-\sqrt{b(j)} \vec{g}(j)\|
$$

$124 b(i)$ is the b-value, and $\vec{g}(i)$ is the diffusion encoding direction. The resulting values in Eq. (1) ranges 125 between $0.6 \sim 0.8$, and a data set would be rejected if the baseline and follow-up scans have a difference 126 in mean Pearson correlation coefficient greater than 0.1. In this study, one ALS patient's data we 127 recruited previously (Abhinav et al., 2014) was rejected because the baseline scan had a substantially 128 lower value due to slice shift during the scan.

The third quality control step was identifying slice-wise signal dropout for each slice in each diffusion-weighted image. This examination excluded slices with a large background region greater than $15 / 16$ of the entire slice area (defined by the brain mask). For each slice, we calculated its Pearson correlation coefficients with four of its signal-related slices, including its upper and lower adjacent slices and the same-location slices of two neighboring diffusion-weighted images defined by Eq. (2). The maximum of these four correlation values was used as the representative correlation coefficient of the slice, and a signal dropout would result in a decrease of this representative correlation coefficient. If a slice had an average decrease of representative correlation coefficient greater than 0.1 in comparison 137 with its four related slices, we identified it as a signal dropout slice. In this study, we accepted the data set if the number of signal dropout slices was smaller than $0.1 \%$ of the total slice number (i.e. $<25$ slices). All data set were screened and passed this criterion.

140 The fourth quality control was checking the b-table orientation using the fiber coherence index (Schilling et al., 2019). One scan was found to have slices order flipped upside down. The slice order and b-table 
142 were corrected before further analysis. The above-mentioned quality control routines are available on

143 DSI Studio (http://dsi-studio.labsolver.org).

144 After the quality control steps, the diffusion data of the follow-up scans were compared with baseline

145 scans using the following analysis:

\section{Empirical distribution of water diffusion}

147 The empirical distribution of water diffusion was calculated from diffusion-weighted signals using 148 generalized q-sampling imaging (GQI)(Yeh et al., 2010). This "empirical distribution" has no assumption 149 of the underlying distribution (e.g. Gaussian distribution), and thus it can be applied to a variety of fiber 150 or biological conditions. The empirical distribution calculated from GQI, termed spin distribution function 151 (SDF), has a different physical definition from the diffusivity calculated from DTI that quantifies how fast 152 the diffusion is. SDF quantifies the accumulated spin density of restricted diffusion sampled at any 153 orientations, and it can be calculated using the formula:

$154 \quad \Psi_{0}(\boldsymbol{r}, \hat{u})=Z_{0} \sum_{i} W_{0}(\boldsymbol{r}, i) \operatorname{sinc}(\sigma \sqrt{6 D \mathrm{~b}(i)}\langle\hat{\mathrm{g}}(i), \hat{\mathrm{u}}\rangle)$

$155 \Psi_{0}(\boldsymbol{r}, \hat{u})$ is the SDF value oriented at $\hat{u}$ and sampled from a voxel located at $\boldsymbol{r} . Z_{0}$ is a scaling constant 156 to convert the arbitrary unit of the diffusion signals to a density unit. $i$ iterates through each diffusion 157 weighted signals $\mathrm{W}(\boldsymbol{r}, \mathrm{i})$, and $\mathrm{b}(\mathrm{i})$ is the $\mathrm{b}$-value, $\hat{\mathrm{g}}(\mathrm{i})$ is the direction of the diffusion sensitization 158 gradient. $\sigma$ is the diffusion sampling ratio controlling the displacement range of the diffusing spins (1.25 159 was used in this study). $D$ is the diffusivity of free water at room temperature.

160 We then calculated the SDFs of the follow-up scan and transformed them into the space of the baseline 161 scan (Fig. 1a and Fig. 1b) so that they could be directly compared. This was done using q-space 
diffeomorphic reconstruction (QSDR)(Yeh and Tseng, 2011), a method that generalized GQI to accept spatial transformation in the reconstruction. QSDR allowed us to simultaneously reconstruct and transform SDF from the follow-up scan to the space of the baseline scan using the following formula:

$$
\Psi_{1}(\boldsymbol{r}, \hat{u})=Z_{1} \sum_{i} W_{1}(\phi(\boldsymbol{r}), i) \operatorname{sinc}(\sigma \sqrt{6 D b(i)}\langle\hat{g}(i), \mathbf{J}(\boldsymbol{r}) \hat{u}\rangle)
$$

where $\phi(\boldsymbol{r})$ transforms spatial coordinate $\boldsymbol{r}$ from the space of the baseline scan to that of follow-up scan. $W_{1}(\phi(\boldsymbol{r}), i)$ is the diffusion-weighted signals at coordinate $\phi(\boldsymbol{r}) . \mathbf{J}(\boldsymbol{r})$ is the Jacobian matrix at the same coordinate that rotates the unit vector $\hat{u}$. There other variables follow the same notations in Eq. (3).

Since the scans were from the same subject, we assumed there was only "rigid body" transformation (i.e. only rotation or translocation) between the scans, and the transformation was a simple matrix-vector multiplication. Please note that this assumption could be violated if there was a large tissue distortion due to edema or tissue removal, and a nonlinear spatial registration should be used in QSDR to handle this problem. The rigid body transformation matrix was obtained by linear registering the b0 images (or the sum of all diffusion-weighted images). We used the negative of the correlation coefficient between

177 The cost function was minimized using a gradient descent method. The rotation matrix of the rigid body 178 transformation was used as the Jacobian for Eq. (4).

Please note that the SDFs calculated from Eq. (3) and (4) have "arbitrary units". Therefore, the $Z_{1}$ 180 constant in Eq. (4) had to be scaled to match the same unit of $Z_{0}$ in Eq. (3). This signal matching was 181 done using the sum of all diffusion-weighted images from two scans: 
183 The isotropic component of an SDF was then removed by subtracting its minimum values.

$184 \Psi_{0}^{\prime}(\boldsymbol{r}, \hat{u})=\Psi_{0}(\boldsymbol{r}, \hat{u})-\min _{\widehat{u}} \Psi_{0}(\boldsymbol{r}, \hat{u})$

185

$\Psi_{1}^{\prime}(\boldsymbol{r}, \hat{u})=\Psi_{1}(\boldsymbol{r}, \hat{u})-\min _{\widehat{u}} \Psi_{1}(\boldsymbol{r}, \hat{u})$

186 Equation (6) and (7) provide the anisotropic component of SDF (termed anisotropy hereafter) to

187 minimize the effect of free water diffusion (Yeh et al., 2013). It is noteworthy that this anisotropy 188 measurement has a different physical meaning from the fractional anisotropy (FA) calculated in DTI. FA 189 is a ratio between 0 and 1 calculated from diffusivities and has no unit. The anisotropic SDF has the 190 same physical unit of the SDF, which is the spin density of diffusing water.

191 Tracking differences in the SDF

192 To track differences along the existing fiber pathways, we first determined the local fiber orientations 193 using the peaks on the sum of $\Psi_{0}(\boldsymbol{r}, \hat{u})$ and $\Psi_{1}(\boldsymbol{r}, \hat{u})$, and then the anisotropy estimated from the 194 summed SDF was used to filter out noisy fibers and to define the termination of the white matter tracks, 195 as what had been done in conventional tractography (Yeh et al., 2013). The percentage difference in the 196 anisotropy between baseline and follow-up scans was then calculated (Fig. 1c):

$197 \quad \Psi_{d}(\boldsymbol{r}, \hat{u})=\frac{2\left(\Psi_{1^{\prime}}(\boldsymbol{r}, \widehat{u})-\Psi_{0^{\prime}}(\boldsymbol{r}, \widehat{u})\right)}{\Psi_{1^{\prime}}(\boldsymbol{r}, \widehat{u})+\Psi_{0^{\prime}}(r, \widehat{u})} \times 100 \%$

198 The percentage changes in the anisotropy, $\Psi_{d}(\boldsymbol{r}, \hat{u})$, can have positive values (blue SDFs in Fig. 1c), 199 which indicates an increase in the density of anisotropic diffusion, or negative values (red SDFs in Fig. 200 1c), which indicates a decrease in the density of anisotropic diffusion.

201 An additional tracking-the-differences criterion was added to the fiber tracking algorithm to track the 202 exact segment with a decrease or an increase in the anisotropy greater than a change threshold, 
203 Specifically, to track pathways with an increase of anisotropy, the additional criterion checked whether

204 the increase of anisotropy was greater than a predefined value of percentage change (e.g. 20\%) and 205 continued tracking as long as the criterion was satisfied:

$206 \Psi_{d}(\boldsymbol{r}, \hat{a})>\theta^{+}$

207 where $\hat{a}$ is the local fiber directions used in the fiber tracking algorithm. Similarly, to track pathways with 208 decreased anisotropy, the criteria continued tracking if the decrease of anisotropy was greater than a 209 predefined value of percentage change (e.g. -20\%):

$210 \Psi_{d}(\boldsymbol{r}, \hat{a})<\theta^{-}$

211 This allowed us to track two different sets of pathways, one for increased anisotropy, one for decreased 212 anisotropy. The other existing criteria in conventional tractography (e.g. seeding strategy, propagation 213 interval, angular threshold, length constraint...etc.) remained in effect as what has been used in the 214 generalized deterministic fiber tracking algorithm (Yeh et al., 2013). It is noteworthy that the angular and 215 anisotropy thresholds in the original tracking algorithm were still used in differential tractography to 216 eliminate noisy fiber and to ensure a correct white matter coverage. The criteria (9) or (10) (termed 217 "positive change threshold" and "negative change threshold") served as additional constraints to limit the 218 findings to the exact segment of pathways with a substantial change in the anisotropy value.

220 In this study, the differential tractogram was obtained by placing a total of $5,000,000$ seeding points in 221 the white matter. The angular threshold was randomly selected between 15 to 90 degrees. The step size 222 was $1 \mathrm{~mm}$, and the anisotropy threshold was automatically determined by DSI Studio. Two iterations of 223 topology-informed pruning (Yeh et al., 2018) were applied to the tractography to remove noisy findings. 
224 The above-mentioned setting was regularly used in conventional tractography. We tested differential 225 tractography with different values of change threshold $(5 \%, 10 \%, 15 \% \ldots 50 \%)$ and length threshold $(5$ $226 \mathrm{~mm}, 10 \mathrm{~mm}, 15 \mathrm{~mm}, \ldots 50 \mathrm{~mm}$ ). Track with lengths shorter than the length threshold was discarded, and

227 the results of different length threshold and change threshold were compared to access its effect on the 228 sensitivity and specificity of differential tractography.

\section{Estimating false discovery rate using a sham setting}

230 Figure 2 illustrates the experimental design that allows for estimating the false discovery rate (FDR) of 231 differential tractography in individual scans. As shown in Fig. 2a, the baseline scan is compared with the 232 follow-up scan (upper row) using differential tractography to reveal tracks with decreased anisotropy (Fig. $2332 \mathrm{~b}$, upper row). If a total of 5,000,000 tracking iterations are conducted, we can view each of them as an 234 independent hypothesis testing since each tracking iteration is done independently. The null hypothesis 235 is "there exists no track with a decrease in the anisotropy". This null hypothesis will be rejected if the 236 track length is longer than a predefined length threshold (e.g. $40 \mathrm{~mm}$ ). Each rejected hypothesis is thus 237 regarded as a "positive finding", but here the finding can be either a true positive or false positive. As 238 shown by the example in the upper row of Fig. 2b, the number of tracks with a length longer than $40 \mathrm{~mm}$ 239 is 13947 , meaning that there are 13947 rejected hypotheses as positive findings. These 13947 findings 240 include true positive and false positive, and we will need to estimate the number of false-positive findings 241 so that FDR can be estimated. To estimate the number of false-positive findings, a sham scan can be 242 acquired on the same day of the baseline scan or within a timeframe that no neuronal change is 243 expected (Fig. 2a, lower row), and thus any positive findings shown in the sham scan should be 244 false-positive findings. The sham scan is also compared with the baseline scan, and the same number 245 of 5,000,000 tracking iterations are conducted to see how many positive findings are generated. The 246 example in the lower row of Fig. $2 \mathrm{~b}$ shows that the sham scan generates a total of 177 tracks with a 
247 length longer than $40 \mathrm{~mm}$, meaning that the estimated number of false-positive findings is 177 . Using 248 this information, we can calculate the FDR, which is $177 / 13947=0.0127$ (Fig. 2c).

\section{Substitute sham}

250 In this retrospective study, we did not have an additional sham scan, and a "substitute sham" approach

251 was used. We assumed that there should be no increased track integrity during the disease course, any 252 findings showing increased anisotropy can be regarded as false-positive findings. Therefore, we can use 253 the number of tracks with increased anisotropy in the follow-up scan as a substitute for the sham scan. 254 Even if tracks with increased integrity do exist due to re-myelination, it will increase our estimated 255 number of false-positive findings. This means that the FDR estimated by this substitute sham approach 256 will be an upper bound of the actual FDR. Therefore, instead of reporting "FDR=0.0127", the FDR 257 calculated using a substitute sham should be reported as "FDR $\leq 0.0127$ ".

258 The processing pipeline for differential tractography and the quality control procedure is implemented in 259 DSI Studio (http://dsi-studio.labsolver.org). Documentation and source code to reproduce the same 260 result is also available on the website.

\section{Results}

\section{Neuronal injury reflected by a decrease of anisotropy}

263 Figure 3a shows the intermediate results of differential tractography applied to an MS patient with optic 264 neuritis (patient \#1, demographics summarized in Table 1). The baseline scan was acquired right after 265 the onset, whereas the follow-up scan was acquired 6 months after. For each fiber orientation in a voxel, 266 differential tractography compares the anisotropy differences between two MRI scans in a common 
267 subject space (Fig. 1a-1c). The fiber orientations with a decrease of anisotropy greater than $30 \%$ could

268 are plotted by red sticks in Fig. 3a. In the figure, most of the differences are distributed near the primary 269 visual pathways, whereas some spurious differences are randomly distributed throughout the entire 270 whiter matter regions, most likely due to local signal variations or registration error.

272 To eliminate these local spurious differences, the "tracking-the-difference" algorithm is applied to the 273 track and link all local differences together into continuous trajectories, and short fragments are 274 discarded using a length threshold of $40 \mathrm{~mm}$ (Fig. 3b). The rationale behind this length threshold is that 275 the local random error does not propagate along fiber pathways, whereas true findings due to neuronal 276 injury will form a continuous decrease of anisotropy along the fiber bundles. A length threshold will 277 effectively differentiate between them and help eliminate false results.

278 The left inset figure in Fig. 3b shows affected tracks in directional colors (red: left-right green: 279 anterior-posterior blue: superior-inferior), whereas the tracks in the right inset figure are color-coded by 280 the percentage decrease of anisotropy suggesting the severity of neuronal injury (yellow: 0\% decrease 281 red: $70 \%$ decrease). In overall, the differential tractogram in Fig. 3b reveals a heterogeneous decrease 282 of anisotropy between $20 \%$ to $50 \%$. All findings are in the bilateral primary visual pathways or their 283 collateral connections. The location of the finding matches well with the patient's medical history of 284 visual field loss in both left and right quadrants. The topology of affected pathways seems to present a 285 ripple effect: not only the primary visual pathway is affected, but also certain collateral connections to the 286 visual cortex has shown a decrease in the anisotropy. Although this patient was fully recovered from the 287 symptoms during the follow-up scan (brief medical history in the Supplementary materials), differential 288 tractography still captures subclinical change near the bilateral optic radiation. 
289 We further compare differential tractography with conventional voxel-wise statistics. Figure 3c shows the 290 axial mapping of anisotropy differences for each voxel using the same data. The red regions are voxels 291 with anisotropy decrease greater than $30 \%$, and the numerous fragments can be observed across the 292 entire brain regions. Those fragments could be due to local random error and thus may not be true 293 findings. This illustrates a typical limitation of a voxel-based imaging biomarker, and there is no pathway 294 information to assist diagnostic evaluation. In comparison, the differential tractogram in Fig. 3b provides 295 track-based imaging biomarkers that can easily assist diagnostic evaluation by offering trajectories 296 information. The findings can be associated with an anatomical pathway to infer the functional 297 correlation.

\section{Conventional tractography versus differential tractography}

299 Figure 4 compares conventional tractography (Fig. 4a) with differential tractography (Fig. 4b) on another 300 MS patient with optic neuritis (patient \#2 demographics summarized in Table 1). The conventional 301 tractography was generated using the baseline scan, whereas the differential tractography was 302 configured to map pathways with more than a 30\% decrease in anisotropy with a length threshold of 40 $303 \mathrm{~mm}$. The trajectories in Fig. 4a and Fig. 4b are both colored-coded with directional colors (red: left-right 304 green: anterior-posterior blue: superior-inferior). The first row shows tractography viewing from the top, 305 whereas the second rows show from the left. The conventional tractography in Fig. 4a visualizes the 306 trajectories of the entire brain fiber pathways, and there is no gross anomaly visible from the 307 tractography that may suggest a major neurological disorder. This is expected because, at the early 308 stage of multiple sclerosis, the patient usually does not present a gross structural change that can be 309 readily identified in conventional tractography. In comparison, the different tractography in Fig. 4b 310 pinpoints the location of affected pathways in the bilateral primary visual pathway near the visual cortex. 
311 The location matches well with the patient's disease presentation of optic neuritis, whereas conventional

312 tractography in Fig. 4a shows no clue to this critical information.

\section{Reliability assessment}

314 We further use patient \#1 and a 42-year-old normal subject as the examples to show how the reliability 315 of differential tractography findings can be quantified using a sham setting. Both the follow-up scans of 316 these two subjects were acquired 6 months after the baseline scans. Figure $5 a$ is the differential

317 tractogram of patient \#1 showing pathways with an increase or decrease anisotropy greater than $30 \%$ at 318 different length thresholds, whereas Fig. $5 \mathrm{~b}$ is the same analysis applied to a healthy subject. Only the 319 decreased anisotropy in patient \#1 (lower row in Fig. 5a) contains possible true findings for neuronal 320 injury, whereas other rows in Fig. 5a and Fig. 5b are all false-positive results due to either physiological 321 noises (cardiovascular or respiratory) or phase distortion artifact. Most false findings can be effectively 322 removed using a longer length threshold, and there is a trade-off between sensitivity and specificity 323 controlled by the length threshold. A longer length threshold renders a more specific result with the 324 expense of losing meaningful findings in shorter segments, whereas a shorter length threshold allows for 325 more findings with a risk of taking false results. To quantify the reliability of findings in the lower row of 326 Fig. 5a, we used the total number of findings at the upper row of Fig. 5a as an estimation for the number 327 of false findings. This assumes that the random error will produce a similar number of false findings in 328 both rows and allows us to estimate FDR.

329 Figure $5 c$ and $5 d$ illustrate the FDR calculation process. Figure $5 c$ lists the number of findings at different 330 length thresholds and the change thresholds for patient \#1 (upper two tables) and the normal subject 331 (lower two tables). For example, a negative change threshold of $30 \%$ means that the tracking algorithm 332 will track only the fiber orientations with a decrease of anisotropy greater than $30 \%$. The tables on the left are numbers of findings with decreased anisotropy, whereas those on the right are numbers of 
334 findings with increased anisotropy. The green colors in the table are those with a larger number of 335 findings, whereas the red colors indicate a smaller number. The tables of the patient show a 336 substantially larger number of findings with decreased anisotropy caused by the disease. In comparison, 337 the two tables of the control subject are substantially similar, and the false results are equally distributed 338 in both tables. This result supports the usage of the "substitute sham" approach mentioned in the 339 Material and Methods section: we can reasonably use the findings with increased anisotropy (the table on the right) of the same subject to estimate the number of false findings for calculating FDR.

341 Figure 5d shows the FDR of patient \#1's findings calculated using the "substitute sham" (the increase of anisotropy results from the same subject). The resulting table shows a trade-off between sensitivity and specificity controlled by both the length threshold and change threshold. A length threshold of $30 \sim 40 \mathrm{~mm}$ and a change threshold at 20 30\% decrease of anisotropy provides us an FDR around 0.01, suggesting that around $1 \%$ of the tracks shown in the differential tractogram are false results. We can use these two thresholds to leverage sensitivity and specificity. For example, lower thresholds are geared toward higher sensitivity to explore potential neuronal injury, whereas higher thresholds can provide a confirmatory answer to the axonal damage. The optimal setting can be different based on the disease condition, scan interval, and purposes (e.g. exploratory or confirmatory).

We further apply differential tractography to patients with different neurological disorders in Fig. 6 and list the FDR of these findings in Table 2. The scan subjects include patients with MS (\#1, \#2), HD (\#3, \#4), ALS (\#5), and epilepsy (\#6). The first three rows show the differential tractograms in three views 354 (left sagittal from left, coronal from the front and axial from top) using directional colors, where the last 355 row shows the differential tractogram with yellow-red colors representing the percentage decrease of anisotropy. 
357 The first notable finding comparing MS patients \#1 and \#2 is that the volume of affected pathways and 358 their decrease of anisotropy reflect the severity of their clinical symptoms. The medical history of patient $359 \quad \# 1$ (Supplementary Materials) indicates a more severe drop in visual acuity to 20/400 in addition to her 360 visual field defect in all quadrants, while patient \#2 only had a decrease of visual acuity to 20/125 with 361 only superior altitudinal visual field defect (Table 1 and Supplementary Materials). The greater severity in 362 patient \#1 is reflected by a larger volume of affected pathways diffusion (patient \#1: $55681.6 \mathrm{~mm}^{3}$, 363 patient \#2: $26124 \mathrm{~mm}^{3}$ ) and a greater decrease of anisotropy shown in the last row. This suggests that 364 differential tractography has a quantitative potential to evaluate disease severity using either the volume 365 of affected tracks or the decrease of their anisotropy.

366 The differential tractogram of HD patients \#3 and \#4 in Fig. 6 both shows affected pathways around the 367 stratum. This matches well with the common understanding that striatal pathways are usually involved in 368 Huntington's disease. Moreover, the differential tractogram in patient \#4 has a greater involvement 369 extending to brainstem and cerebellum, suggesting a worse motor performance. This seems to match 370 the patient's medical history of a higher motor score of 64 (Table 1 and Supplementary Materials). The 371 patient also had more asymmetric dystonia, matching the asymmetry presentation of the differential 372 tractography.

373 Patient \#5 in Fig. 6 is an ALS patient. It is noteworthy that this patient had mostly lower motor neuron 374 symptoms (weakness), and thus might not have positive findings in the brain. The differential tractogram 375 of this patient were obtained using a $15 \%$ negative change threshold because a threshold of $30 \%$ 376 yielded no findings. Differential tractography reveals only a minor decrease in this patient (other cases in 377 Fig. 6 have mostly greater than $30 \%$ decrease). This could be explained by the fact that the patient had 378 predominately lower motor neuron symptoms affecting predominately peripheral nerves. Therefore, the 379 findings in the central nervous system could be only subclinical. Nonetheless, as we lowered the change 
threshold to $15 \%$, differential tractography showed affected pathways in the right lower corticospinal pathway (blue-purple colored), superior cerebellar peduncle, and posterior corpus callosum, as shown in

Fig. 6. The right corticospinal pathway involvement seems to match the patient's history of left side involvement, but it is noteworthy that the FDR of these findings were much higher (FDR 0.2 in Table 2), meaning that around $1 / 5$ of the findings are false positives. The corpus callosum and occipital lobe findings could be subclinical damage and did not present any clinical symptoms (to be discussed in the Discussion section).

Patient \#6 was a 51-year-old male with right anterior lobectomy. He was previously an epileptic patient with recurrent epilepsy (Supplementary Materials). The MRI scans were done before the surgery and one year after the surgery. Differential tractography accurately locates the location of the surgical resection in the mesial structures and approximately $5 \mathrm{~cm}$ of the anterior temporal neocortex. Moreover, it further reveals the pathways that were affected by the resection. While the surgical resection only removed part of the temporal gyri, the affected pathways involve much greater connection networks. Furthermore, the last row shows that the decrease of anisotropy is mostly greater than $50 \%$, indicating a greater axonal loss due to the surgical removal of the brain tissue.

The last column in Fig. 6 shows the differential tractogram of a control. We applied the same settings to examine how differential tractography may capture false results. The result shows a mild decrease of anisotropy as presented by yellow tracks in the last row, a clue that the change may be a false positive result. Furthermore, there are only 74 findings located at the prefrontal cortex, and these findings are relatively insignificant compared to those of the patient population that shows thousands of findings (Table 2). Moreover, the location of the findings is known to be heavily affected by the phase distortion 401 artifact, and the findings could be due to the different level of distortion between the repeat scans. 
403 with increase anisotropy for this control subject. This result suggests that the FDR estimation still have

404 its limitation if the number of findings is sufficiently small. The interpretation of differential tractography 405 results still needs to consider the percentage decrease of anisotropy, the total number of findings, and 406 possible sources of imaging artifact.

407 In general, the findings in Fig. 6 allows us to quickly differentiates the possible locations of neuronal 408 injury and evaluate the severity. The affected pathways in MS, HD, and ALS patients show distinctly 409 different topology, allowing for differential diagnosis or prognosis evaluation. Table 2 further shows how 410 we can use the sham setting to calculate FDR to evaluate the reliability of the results.

\section{Performance difference after dropping high b-value acquisitions}

412 We further investigate whether high b-value signals contribute to the detection power of differential 413 tractography. In this study, we acquired $22 \mathrm{~b}$-values ranging from 0 to $7,000 \mathrm{~s} / \mathrm{mm}^{2}$, but most diffusion 414 MRI studies would acquire b-values between 1,000 and $3,000 \mathrm{~s} / \mathrm{mm}^{2}$ because higher b-values result in 415 poorer signal-to-noise (SNR) ratio. This can be observed in Fig. 7a showing the diffusion-weighted 416 images at different b-values from patient \#1. It is thus of a practical consideration to limit the b-values 417 under a certain maximum value. To examine whether the high b-value acquisition is necessary for 418 differential tractography, we repeated the same differential tractography analysis but used only the 419 signals with b-values between 0 and $3,000 \mathrm{~s} / \mathrm{mm}^{2}$ (a total of 85 sampling directions). The reduced dataset is very similar to the scheme acquired in a previous study (Wang et al., 2011).

421 Fig. 7b shows a qualitative comparison on subject \#1 before and after dropping high b-value signals.

422 The location of the findings is mostly consistent; however, dropping high b-values results in substantial 423 loss of the findings (annotated by the red circle). This implies that certain stages of neuronal injury can 424 only be detected by very high b-values. The quantitative comparison is listed in Table 3 . As shown in the 
425 table, there is a unanimous drop in positive findings for all patients, ranging from $38 \%$ decrease to $97 \%$ 426 decrease, while the false findings in the normal subject increased by 7 times. Consequently, patients \#1 427 and \#3 have a dramatic FDR increase from less than 0.05 to more than 0.3 , making the findings not 428 statistically reliable. It seems that differential tractography using only low b-value signals is more 429 sensitive to physiological variations, whereas differential tractography using both high and low b-value 430 signals are more reliable in detecting are more specific to neuronal injury. This could be explained by the 431 fact that the low b-value images in Fig. 7a have more signal contribution from free water diffusion in 432 ventricles and subarachnoid space, whereas high b-value images only have signals from restricted 433 diffusion in the core white matter. Excluding high b-value signals will shift the focus to free water 434 diffusion and lead to more false-positive results. The overall result supports the necessity of including 435 high b-value acquisition for differential tractography to detect neuronal injury.

\section{Discussion}

437 Here we report a novel tractography method to reveal fiber pathways affected by a neuronal injury. We

438 found that differential tractography could serve as a track-based biomarker to provide localization of 439 neuronal injury and allow for quantifying its severity using the decrease of anisotropy and the total 440 volume of affected pathways. The estimated FDR further offered reliability information to interpret the 441 results, and the findings correlated well with clinical presentations of each individual.

\section{Comparison with other methods}

443 Diffusion MRI fiber tracking can be viewed as a clustering process that is directionally sensitive. By using 444 spatial relations across multiples voxels, the tracking-the-difference strategy used in differential 
445 tractography has the potential to differentiate true findings from local errors, since neuronal injury will 446 propagate along axonal fibers while local error stays locally. This tracking-the-difference strategy is 447 conceptually similar to clustering used in voxel-based morphometry (Ashburner and Friston, 2000) or 448 fMRI studies (Woo et al., 2014), which groups voxel-wise statistics into clusters to achieve greater 449 statistical power. The difference is that the clustering used in previous studies did not consider local fiber 450 orientations and would include voxels at all possible neighboring directions, whereas differential 451 tractography only allows findings along the fiber pathways. This improvement is similar to those using 452 fiber geometry to increase specificity (Raffelt et al., 2017; Zhang et al., 2018), and It is likely that this 453 structural restriction may achieve a better specificity than a regular clustering approach.

\section{Optimal b-table for differential tractography}

455 The diffusion MRI acquisitions played a critical role to boost the sensitivity of differential tractography.

456 Our result shows that acquisitions using only b-values lower than $3,000 \mathrm{~s} / \mathrm{mm}^{2}$ may have a limited 457 detection power for early neuronal injury. We also tested differential tractography on existing DTI data, 458 and the preliminary result (not reported here) also showed a substantially higher rate of false findings.

459 This is not surprising because a typical DTI acquisition only acquires only one b-value of 1,000 2,000 $460 \mathrm{~s} / \mathrm{mm}^{2}$ at 30 60 directions, whereas in this study, we acquired $22 \mathrm{~b}$-values from 0 to $7,000 \mathrm{~s} / \mathrm{mm}^{2}$ at 257 461 directions. It is likely that early axonal injury affects mostly restricted diffusion and can only be reliably 462 captured if a wider range of b-value is acquired with enough diffusion sampling directions.

463 Multishell-acquisition could also be used by differential tractography to detect neuronal injury, but its 464 challenges are how to ensure a homogeneous sampling density in the q-space acquisition to make the 465 acquisition "rotation invariant". In addition, most multi-shell acquisitions have an over-sampling problem 466 within each shell and under-sampling problem between shells, which can be observed by plotting the 
467 sampling points in the q-space. Consequently, the inter-shell coverage may not be sufficient enough to 468 capture a variety of diffusion patterns, while the intra-shell signals can be easily interpolated due to 469 oversampling, meaning that there is redundancy which can be further reduced to save scanning time.

470 The q-space grid sampling used in this study seems to the method of choice for differential tractography 471 because it has uniform sampling density in the q-space and covers 22 different b-values. This 472 maximized the chance to detect a variety of diffusion pattern that could be altered during the disease 473 process. Q-space imaging used to be criticized for its lengthy scanning time; however, after the 474 introduction of the multi-band sequences, the updated q-space grid sampling scheme could be acquired 475 within 12 minutes for 256 directions and 6 minutes for 128 directions, making it highly feasible for clinical 476 studies. The exact steps to reproduce these two q-space grid acquisitions are documented on the DSI 477 Studio website (http://dsi-studio.labsolver.org/).

\section{Anisotropy measurement for differential tractography}

479 The anisotropy used in this study is not the commonly used FA provided by DTI. FA is a voxel-wise 480 measurement, and it does not selectively quantify the anisotropy at different fiber population within the 481 same voxel. In comparison, the anisotropy in this study can quantify the anisotropy for each fiber 482 population at different directions This results in greater specificity to individual's connectivity patterns 483 (Yeh et al., 2016) and better performance in handling the partial volume effect (Yeh et al., 2013).

\section{Subclinical findings}

485 Another interesting result we observed in this study is that differential tractography seems to capture 486 subclinical findings: certain pathways could be injured without any obvious clinical symptoms reported 487 by patients. For example, the two MS patients shown in Fig. 5 were symptom-free during the follow-up 
scans after the steroid treatments (Supplementary Materials); however. differential tractography still captures a substantial number of findings related to the primary visual pathways, and the decrease of the anisotropy diffusion correlates well with the severity of the initial clinical presentation. Similarly, by lowering the detection threshold, the ALS patient in Fig. 5 also shows substantial involvement in the posterior corpus callosum that connects to the occipital lobe. Although there is no clinical presentation of this patient associated with the findings, subclinical callosal damage for ALS patients is not uncommon 494 (Filippini et al., 2010), and there are also studies suggested subclinical involvement of occipital lobe in 495 the ALS patients (Loewe et al., 2017; Zhang et al., 2017). The ability to capture subclinical findings has a 496 profound clinical implication. It suggests that differential tractography is sensitive enough to provide 497 additional evaluation value on top of existing clinical scales and scores. This may facilitate the 498 development of new treatment to target early subclinical change that may end up with irreversible 499 damage.

\section{Limitations and possible pitfalls}

501 There are limitations in differential tractography. Differential tractography only works on longitudinal 502 scans of the same subject and only reflects the change of anisotropy within the time frame of the repeat 503 scans. It cannot access track integrity in a cross-sectional setting, nor does it able to detect any 504 abnormality or axonal injury prior to the baseline scans. Furthermore, differential tractography still has 505 false results if the artifact also propagates coincidentally along a fiber pathway. The parallel imaging or 506 eddy current artifact often gives rise to straight lines near the brain surface but may appear like a 507 spuriously legitimate connection. Misalignment between baseline and follow-up scans can also generate 508 a false result, and it can happen due to registration error or brain tissue shift after surgical intervention. 
509 There are still other possible causes of false results. The subjects may have substantial movement in

510 the follow-up scan, but not in the study or sham scan. There may be inconsistency in image acquisition

511 between repeat scans such as changing the head coils or scanning protocol. Both scenarios can

512 produce spurious findings, and thus a series of quality control is always needed to avoid these getting

513 false results. Furthermore, the findings in differential tractography still need to be validated against

514 neuroanatomy. Spurious findings often appear near the brain surface with odd trajectories (straight

515 lines), while true findings tend to follow the trajectories of well-known neuroanatomical pathways. Prior

516 neuroanatomy knowledge may help exclude false results from true findings.

517 There are also limitations in the reliability assessment. The sham setting in this study uses only one scan,

518 and thus the calculated FDR only considered false results due to local random error (e.g. noise which

519 randomly occurs at each imaging voxel) and does not include those due to systematic errors (errors that

520 affects all imaging voxels simultaneously) such as subject movements, coil quality, or signal drift. To

521 detect these systematic errors, we introduced four quality-control routines in the Material and Methods

522 section to discard scans that had a substantial amount of systematic errors. Moreover, we did not

523 acquire an additional sham scan in this study and used the "substitute sham" approach. The substitute

524 sham approach can overestimate the number of false results due to a genuine increase of anisotropy

525 (e.g. re-myelination after recovering from a neuronal injury). The consequence is that the FDR could be

526 higher than the actual value, and the reliability of the findings could be underestimated, leading to a risk

527 of missing meaningful findings.

528 Last, the diffusion MRI protocol in this study could be further optimized. Our result showed that high 529 b-value played an important role in boosting the sensitivity of differential tractography, but the optimal 530 value range still needs further investigation. Moreover, this study did not utilize the recent multi-band 531 acquisition to reduce the scanning time, and future studies will utilize multi-band sequences to make 
532 differential tractography more feasible for clinical applications (e.g. 6-minutes grid-128 and 12-minute 533 grid-256 acquisitions mentioned in the previous paragraphs).

\section{Clinical applications}

535 Differential tractography can be used in differential diagnosis or prognostic evaluation after treatment or 536 intervention. Neurologists can use it to differentiate the cause of a neurological disorder as patients with 537 different neurological disorders will present distinctly different spatial patterns in their affected pathways. 538 The location will provide a clue about the possible causes to resolve difficult clinical cases. This is 539 otherwise not achievable in structural MRI unless a gross lesion or atrophy is visible in the late stage of 540 neuronal injury. Another application of differential tractography is for evaluating an intervention or 541 treatment. Differential tractography can provide an objective quantitation that is directly comparable 542 across subjects and less susceptible to observer differences. It could minimize variance due to evaluator 543 differences and increase effect size in comparison with the conventional evaluation conducted by 544 patients or neurologists. This opens a gate for early treatments to restore subclinical injuries before 545 those injuries accumulate to become a major functional deficit.

\section{Acknowledgments}

547 The research reported in this publication was partly supported by NIMH of the National Institutes of 548 Health under award number R56MH113634. The content is solely the responsibility of the authors and 549 does not necessarily represent the official views of the National Institutes of Health. The MRI scans were 550 partly supported by the Walter L. Copeland Fund of The Pittsburgh Foundation. 


\section{References}

552

553

554

555

556

557

558

559

560

561

562

563

564

565

566

567

568

569

570

571

572

573

574

575

576

577

578

579

580

581

582

583

Abhinav, K., Yeh, F.C., El-Dokla, A., Ferrando, L.M., Chang, Y.F., Lacomis, D., Friedlander, R.M., Fernandez-Miranda, J.C., 2014. Use of diffusion spectrum imaging in preliminary longitudinal evaluation of amyotrophic lateral sclerosis: development of an imaging biomarker. Front Hum Neurosci $8,270$.

Ashburner, J., Friston, K.J., 2000. Voxel-based morphometry--the methods. Neuroimage 11, 805-821. Basser, P.J., Mattiello, J., LeBihan, D., 1994. Estimation of the effective self-diffusion tensor from the NMR spin echo. J Magn Reson B 103, 247-254.

Budde, M.D., Xie, M., Cross, A.H., Song, S.K., 2009. Axial diffusivity is the primary correlate of axonal injury in the experimental autoimmune encephalomyelitis spinal cord: a quantitative pixelwise analysis. J Neurosci 29, 2805-2813.

Callaghan, P.T., 1991. Principles of Nuclear Magnetic Resonance Microscopy. Oxford University Press. Filippini, N., Douaud, G., Mackay, C.E., Knight, S., Talbot, K., Turner, M.R., 2010. Corpus callosum involvement is a consistent feature of amyotrophic lateral sclerosis. Neurology 75, 1645-1652.

Glasser, M.F., Smith, S.M., Marcus, D.S., Andersson, J.L., Auerbach, E.J., Behrens, T.E., Coalson, T.S., Harms, M.P., Jenkinson, M., Moeller, S., Robinson, E.C., Sotiropoulos, S.N., Xu, J., Yacoub, E., Ugurbil, K., Van Essen, D.C., 2016. The Human Connectome Project's neuroimaging approach. Nat Neurosci 19, 1175-1187.

Henf, J., Grothe, M.J., Brueggen, K., Teipel, S., Dyrba, M., 2018. Mean diffusivity in cortical gray matter in Alzheimer's disease: The importance of partial volume correction. Neuroimage Clin 17, 579-586.

Huisman, T.A., Schwamm, L.H., Schaefer, P.W., Koroshetz, W.J., Shetty-Alva, N., Ozsunar, Y., Wu, O., Sorensen, A.G., 2004. Diffusion tensor imaging as potential biomarker of white matter injury in diffuse axonal injury. AJNR Am J Neuroradiol 25, 370-376.

Loewe, K., Machts, J., Kaufmann, J., Petri, S., Heinze, H.J., Borgelt, C., Harris, J.A., Vielhaber, S., Schoenfeld, M.A., 2017. Widespread temporo-occipital lobe dysfunction in amyotrophic lateral sclerosis. Sci Rep 7, 40252.

Maier-Hein, K.H., Neher, P.F., Houde, J.C., Cote, M.A., Garyfallidis, E., Zhong, J., Chamberland, M., Yeh, F.C., Lin, Y.C., Ji, Q., Reddick, W.E., Glass, J.O., Chen, D.Q., Feng, Y., Gao, C., Wu, Y., Ma, J., Renjie, H., Li, Q., Westin, C.F., Deslauriers-Gauthier, S., Gonzalez, J.O.O., Paquette, M., St-Jean, S., Girard, G., Rheault, F., Sidhu, J., Tax, C.M.W., Guo, F., Mesri, H.Y., David, S., Froeling, M., Heemskerk, A.M., Leemans, A., Bore, A., Pinsard, B., Bedetti, C., Desrosiers, M., Brambati, S., Doyon, J., Sarica, A., Vasta, R., Cerasa, A., Quattrone, A., Yeatman, J., Khan, A.R., Hodges, W., Alexander, S., 
Romascano, D., Barakovic, M., Auria, A., Esteban, O., Lemkaddem, A., Thiran, J.P., Cetingul, H.E., Odry, B.L., Mailhe, B., Nadar, M.S., Pizzagalli, F., Prasad, G., Villalon-Reina, J.E., Galvis, J., Thompson, P.M., Requejo, F.S., Laguna, P.L., Lacerda, L.M., Barrett, R., Dell'Acqua, F., Catani, M., Petit, L., Caruyer, E., Daducci, A., Dyrby, T.B., Holland-Letz, T., Hilgetag, C.C., Stieltjes, B., Descoteaux, M., 2017. The challenge of mapping the human connectome based on diffusion tractography. Nat Commun 8, 1349. Melonakos, E.D., Shenton, M.E., Rathi, Y., Terry, D.P., Bouix, S., Kubicki, M., 2011. Voxel-based morphometry (VBM) studies in schizophrenia-can white matter changes be reliably detected with VBM? Psychiatry Res 193, 65-70.

Raffelt, D.A., Tournier, J.D., Smith, R.E., Vaughan, D.N., Jackson, G., Ridgway, G.R., Connelly, A., 2017. Investigating white matter fibre density and morphology using fixel-based analysis. Neuroimage 144, 58-73.

Rovira, A., Wattjes, M.P., Tintore, M., Tur, C., Yousry, T.A., Sormani, M.P., De Stefano, N., Filippi, M., Auger, C., Rocca, M.A., Barkhof, F., Fazekas, F., Kappos, L., Polman, C., Miller, D., Montalban, X., group, M.s., 2015. Evidence-based guidelines: MAGNIMS consensus guidelines on the use of MRI in multiple sclerosis-clinical implementation in the diagnostic process. Nat Rev Neurol 11, 471-482. Schilling, K.G., Yeh, F.C., Nath, V., Hansen, C., Williams, O., Resnick, S., Anderson, A.W., Landman, B.A., 2019. A fiber coherence index for quality control of B-table orientation in diffusion MRI scans. Magn Reson Imaging 58, 82-89. Song, S.K., Sun, S.W., Ramsbottom, M.J., Chang, C., Russell, J., Cross, A.H., 2002. Dysmyelination revealed through MRI as increased radial (but unchanged axial) diffusion of water. Neuroimage 17, 1429-1436.

Song, S.K., Yoshino, J., Le, T.Q., Lin, S.J., Sun, S.W., Cross, A.H., Armstrong, R.C., 2005. Demyelination increases radial diffusivity in corpus callosum of mouse brain. Neuroimage 26, 132-140. Tabrizi, S.J., Langbehn, D.R., Leavitt, B.R., Roos, R.A., Durr, A., Craufurd, D., Kennard, C., Hicks, S.L., Fox, N.C., Scahill, R.I., Borowsky, B., Tobin, A.J., Rosas, H.D., Johnson, H., Reilmann, R., Landwehrmeyer, B., Stout, J.C., investigators, T.-H., 2009. Biological and clinical manifestations of 611 Huntington's disease in the longitudinal TRACK-HD study: cross-sectional analysis of baseline data. 612 Lancet Neurol 8, 791-801.

613 Tabrizi, S.J., Reilmann, R., Roos, R.A., Durr, A., Leavitt, B., Owen, G., Jones, R., Johnson, H., 614 Craufurd, D., Hicks, S.L., Kennard, C., Landwehrmeyer, B., Stout, J.C., Borowsky, B., Scahill, R.I., 615 Frost, C., Langbehn, D.R., investigators, T.-H., 2012. Potential endpoints for clinical trials in 616 premanifest and early Huntington's disease in the TRACK-HD study: analysis of 24 month 617 observational data. Lancet Neurol 11, 42-53. 
Wang, Y., Wang, Q., Haldar, J.P., Yeh, F.C., Xie, M., Sun, P., Tu, T.W., Trinkaus, K., Klein, R.S., Cross, A.H., Song, S.K., 2011. Quantification of increased cellularity during inflammatory demyelination. Brain 134, 3590-3601. Wattjes, M.P., Rovira, A., Miller, D., Yousry, T.A., Sormani, M.P., de Stefano, M.P., Tintore, M., Auger, C., Tur, C., Filippi, M., Rocca, M.A., Fazekas, F., Kappos, L., Polman, C., Frederik, B., Xavier, M., group, M.s., 2015. Evidence-based guidelines: MAGNIMS consensus guidelines on the use of MRI in multiple sclerosis--establishing disease prognosis and monitoring patients. Nat Rev Neurol 11, 597-606.

Wedeen, V.J., Rosene, D.L., Wang, R., Dai, G., Mortazavi, F., Hagmann, P., Kaas, J.H., Tseng, W.Y., 2012. The geometric structure of the brain fiber pathways. Science 335, 1628-1634.

Werring, D.J., Clark, C.A., Barker, G.J., Thompson, A.J., Miller, D.H., 1999. Diffusion tensor imaging of lesions and normal-appearing white matter in multiple sclerosis. Neurology 52, 1626-1632. Diffusion tensor imaging can detect and quantify corticospinal tract degeneration after stroke. J Neurol Neurosurg Psychiatry 69, 269-272.

Woo, C.W., Krishnan, A., Wager, T.D., 2014. Cluster-extent based thresholding in fMRI analyses: pitfalls and recommendations. Neuroimage 91, 412-419. Yeh, F.C., Panesar, S., Barrios, J., Fernandes, D., Abhinav, K., Meola, A., Fernandez-Miranda, J.C., 2018. Automatic Removal of False Connections in Diffusion MRI Tractography Using Topology-Informed Pruning (TIP). Neurotherapeutics. Yeh, F.C., Tseng, W.Y., 2011. NTU-90: a high angular resolution brain atlas constructed by q-space diffeomorphic reconstruction. Neuroimage 58, 91-99. diffusion fiber tracking improved by quantitative anisotropy. PLoS ONE 8, e80713. Yeh, F.C., Vettel, J.M., Singh, A., Poczos, B., Grafton, S.T., Erickson, K.I., Tseng, W.I., Verstynen, T.D., 2016. Quantifying Differences and Similarities in Whole-Brain White Matter Architecture Using

644 Local Connectome Fingerprints. PLoS Comput Biol 12, e1005203. Yeh, F.C., Wedeen, V.J., Tseng, W.Y., 2010. Generalized q-sampling imaging. IEEE Trans Med Imaging 29, 1626-1635. Cai, W., Rathi, Y., O'Donnell, L.J., 2018. Suprathreshold fiber cluster statistics: Leveraging white matter geometry to enhance tractography statistical analysis. Neuroimage 171, 341-354. Zhang, Y., Fang, T., Wang, Y., Guo, X., Alarefi, A., Wang, J., Jiang, T., Zhang, J., 2017. Occipital cortical gyrification reductions associate with decreased functional connectivity in amyotrophic lateral sclerosis. Brain Imaging Behav 11, 1-7. 
a

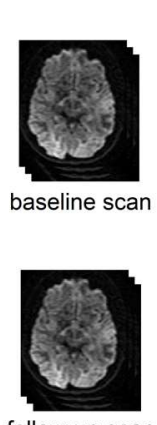

follow-up scan b

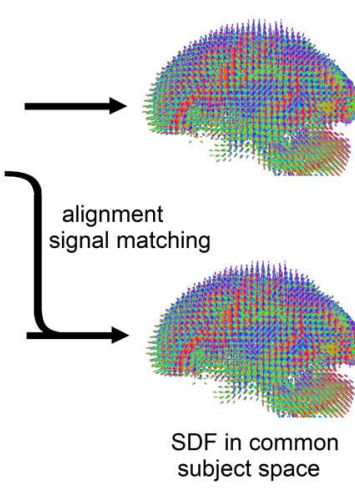

SDF in common
C

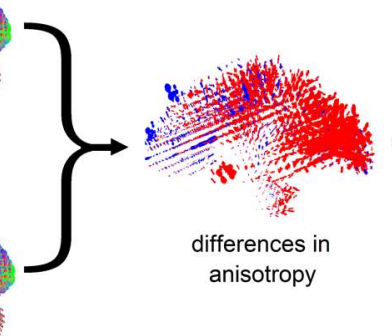

d

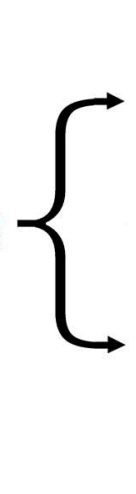

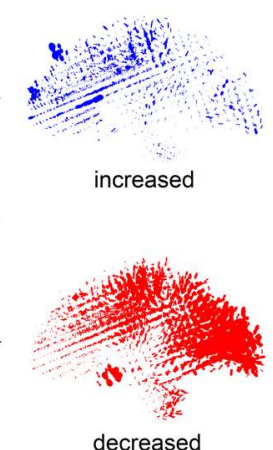

decreased e
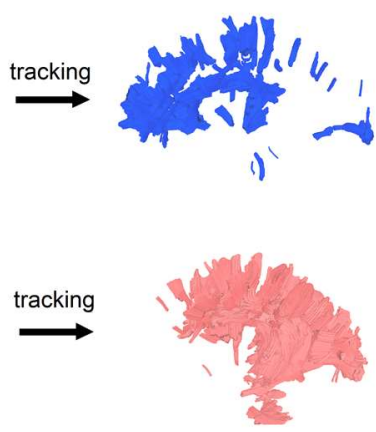

differential tractography

655 Fig. 1

656 The flow chart of differential tractography. (a) The baseline and follow-up scans of the same subject are

657 spatially aligned, and the diffusion signals are scaled to the same unit. (b) The spin distribution function

658 (SDF) from two scans are reconstructed in the same common subject space. (c) The difference in the

659 anisotropic component of SDF is computed for each fiber orientation. (d) Increased and decreased

660 anisotropy values are separated to guide a "tracking-the-difference" algorithm. (e) Differential

661 tractography shows the exact segment of tracks with increased and decreased anisotropy, respectively.

662 The tracks with decreased anisotropy suggest possible neuronal injury, whereas the number of tracks

663 with increased anisotropy can be used to estimate the number of false findings. 


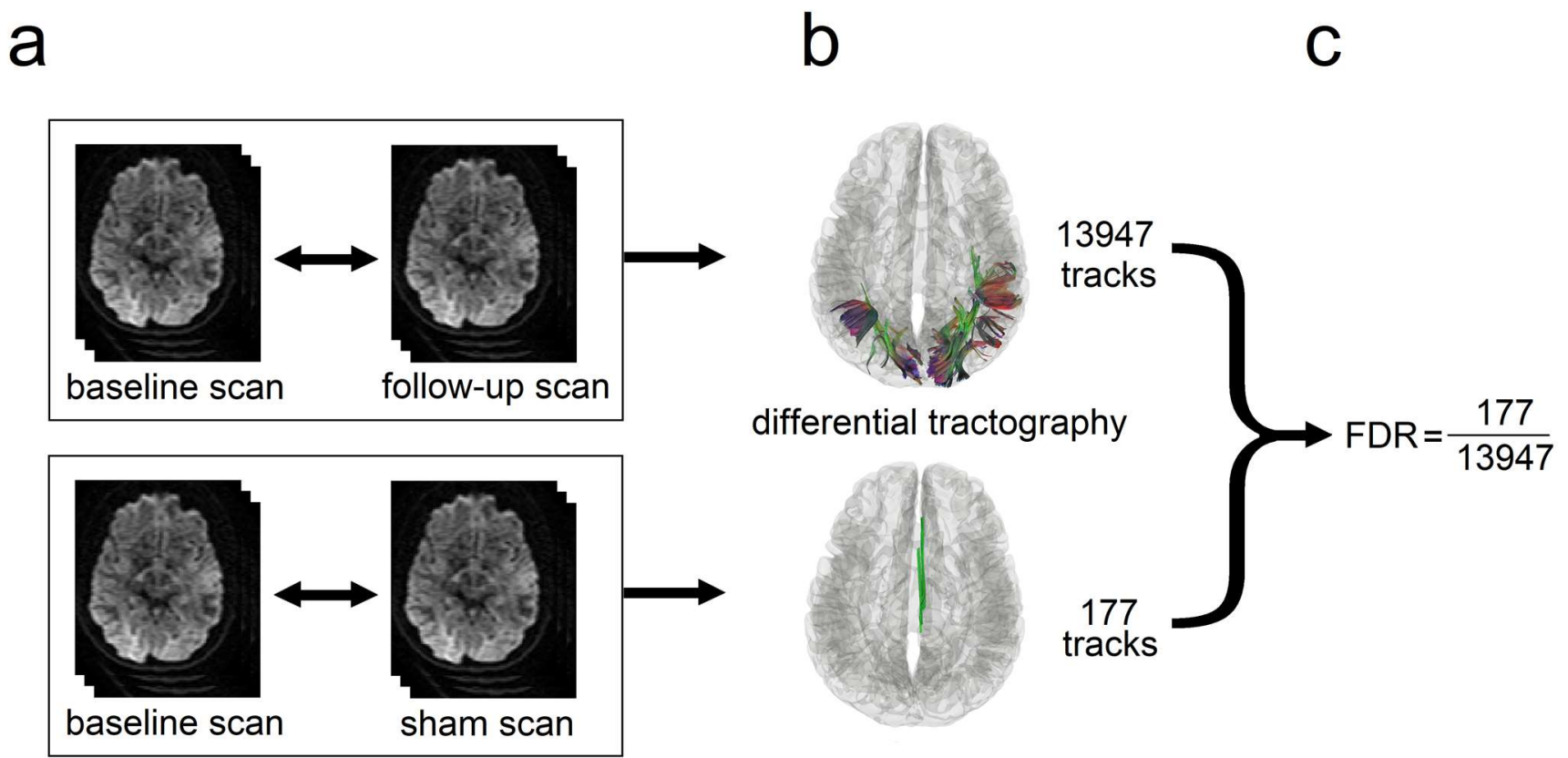

Fig. 2

667 Diagram showing the sham setting for calculating the false discovery rate (FDR). (a) The baseline scan 668 is compared with a follow-up scan (upper row) and a sham scan (lower row), respectively. The follow-up 669 scan is often acquired months after the baseline scan to capture the true positive findings, whereas the 670 sham scan can be a repeat scan on the same day or any scan setting that ensures the findings are all 671 false positives. (b) The number of findings from the follow-up scan (upper row) includes both true and 672 false positives, whereas the number from the sham scan (lower row) include only false-positive findings. 673 (c) These two numbers can be used to estimate FDR. 
a

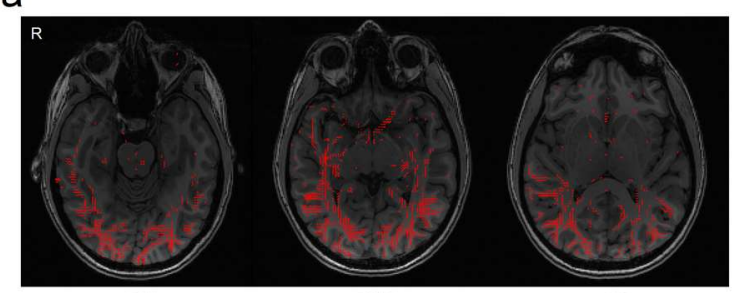

b
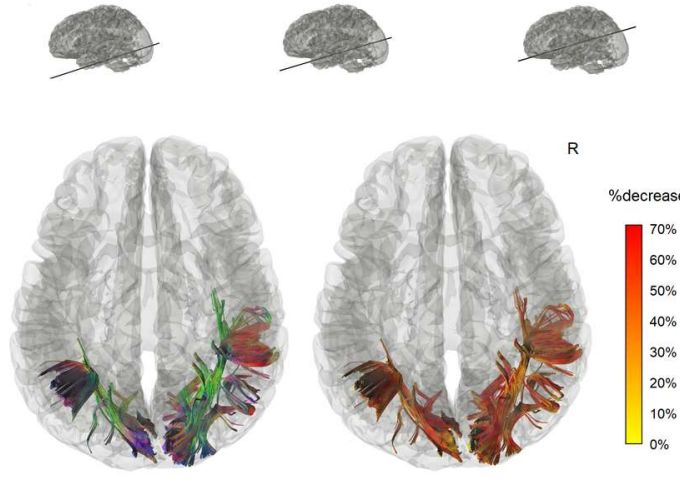

C

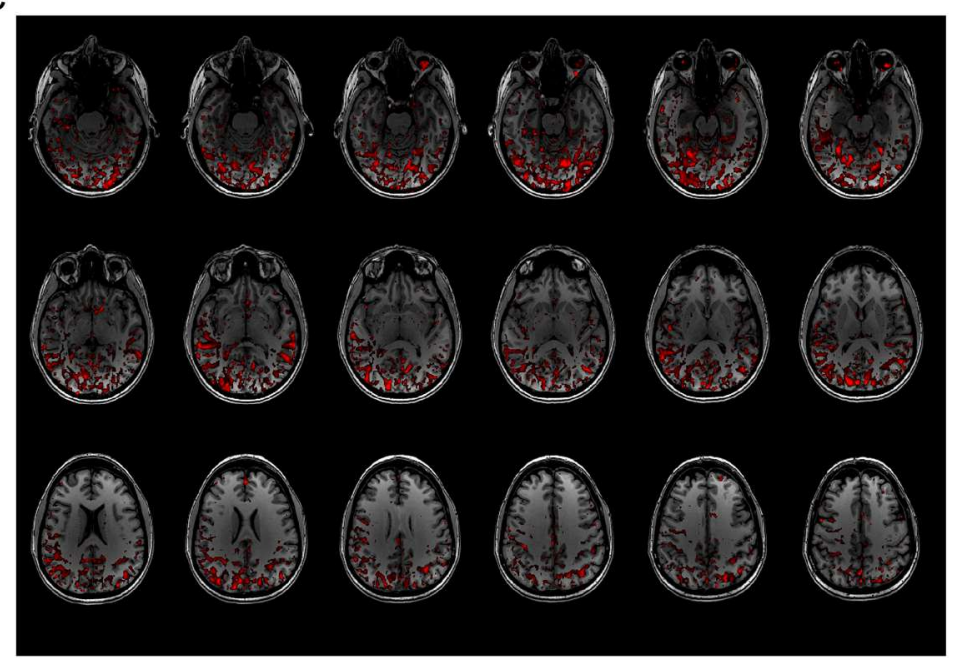

Fig. 3

677 Differential tractography of a multiple sclerosis patient with the first episode of optic neuritis. (a) The

678 intermediate result of differential tractography shows red sticks indicating local fiber orientations with a

679 negative change threshold of $30 \%$ between repeat scans. The sticks are mostly distributed along the

680 primary visual pathways, while sporadic false findings can also be found throughout the entire whiter

681 matter regions due to local signal variations. (b) The red sticks are tracked and connected into

682 continuous trajectories, whereas the other unaffected parts of the white matter pathways are ignored.

683 The resulting 3D presentation is the differential tractogram of the patient showing the exact segment of

684 pathways with a substantial decrease in anisotropy. The tractography can be rendered by directional

685 colors (left) or severity-coded color (right) to provide information about the spatial location, and the

686 severity of the axonal damage can be quantified by percentage decrease of anisotropic diffusion. (c) The

687 same data analyzed by voxel-based differences show numerous fragmented findings possibly due to

688 numerous local random error. There is no track information to assist correlating structure with a function and differentiating true findings from false ones. 
bioRxiv preprint doi: https://doi.org/10.1101/576025; this version posted July 16,2019 . The copyright holder for this preprint (which was not certified by peer review) is the author/funder, who has granted bioRxiv a license to display the preprint in perpetuity. It is made available under 


\section{a}

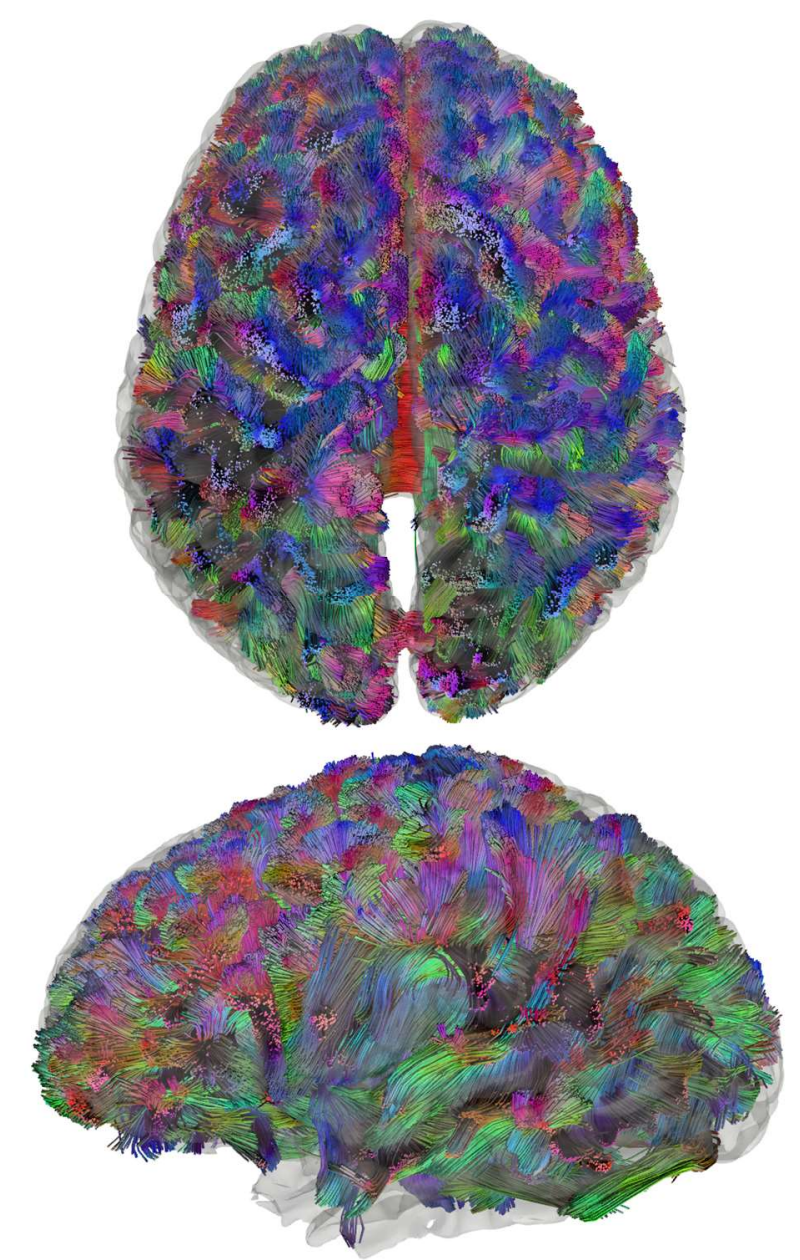

\section{b}
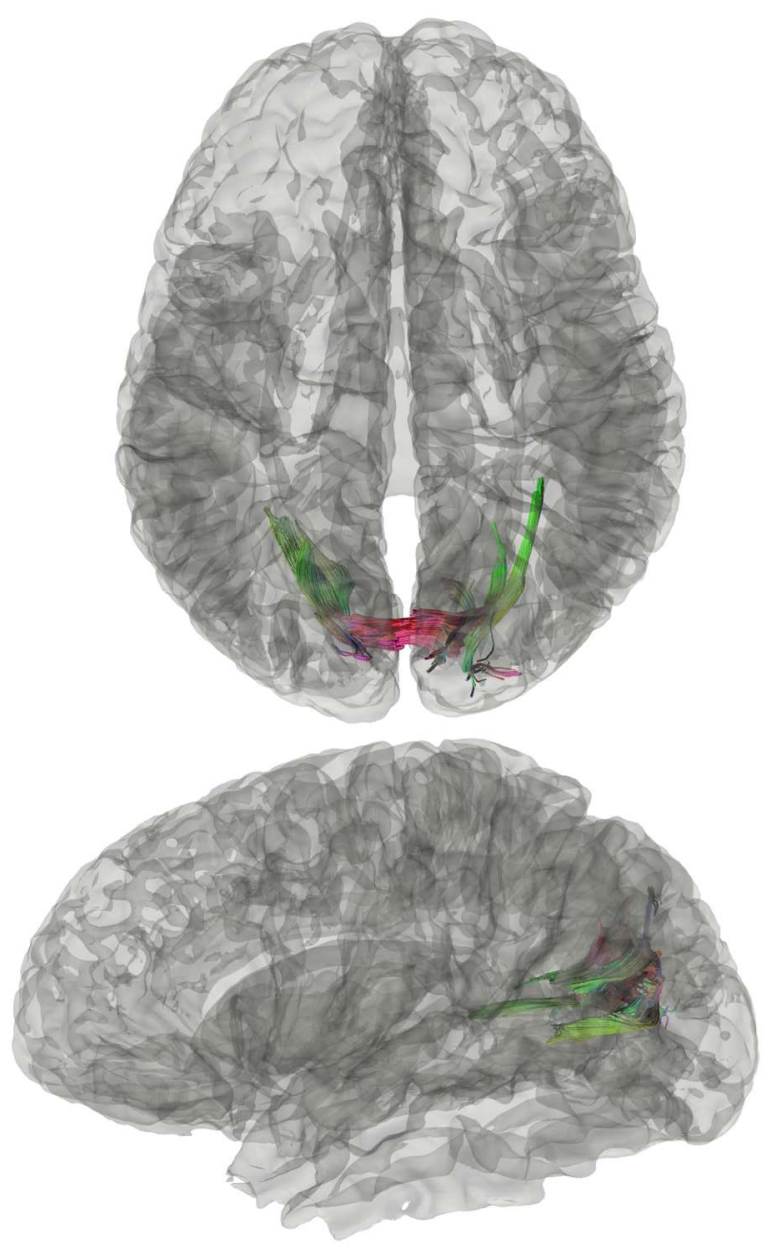

692 Fig. 4

693 Conventional tractography compared with differential tractography on a multiple sclerosis patient with

694 the first episode of optic neuritis. (a) Conventional tractography shows all existing fiber pathways in the

695 human brain and is insensitive to any subtle decrease in the diffusion property. (b) Differential

696 tractography ignores unaffected regions and shows the exact segments of the pathways that have a

697 substantial decrease of anisotropy quantified between repeat scans of the same individual. 
bioRxiv preprint doi: https://doi.org/10.1101/576025; this version posted July 16, 2019. The copyright holder for this preprint (which was not certified by peer review) is the author/funder, who has granted bioRxiv a license to display the preprint in perpetuity. It is made available under aCC-BY 4.0 International license.

a

length threshold

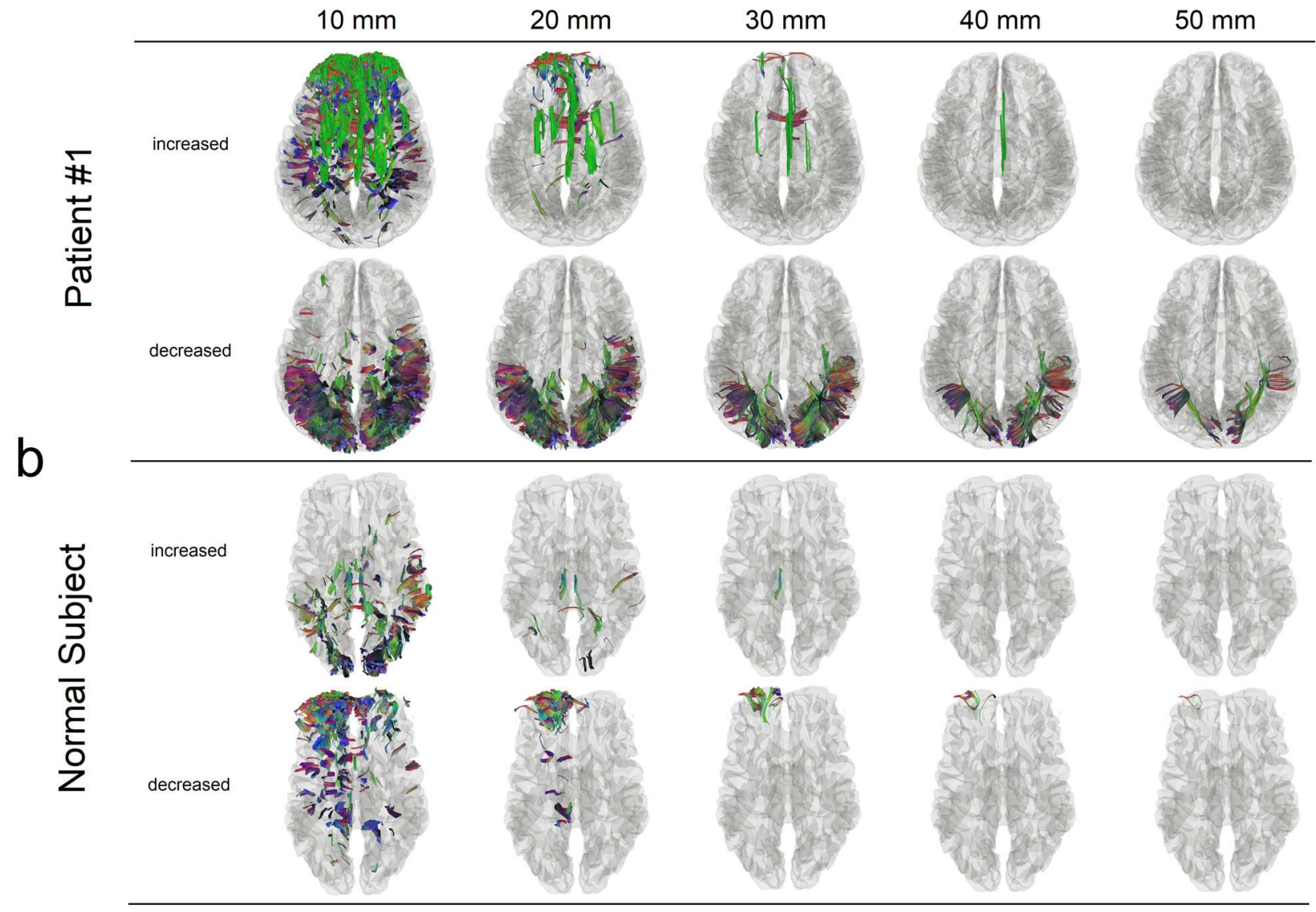

C
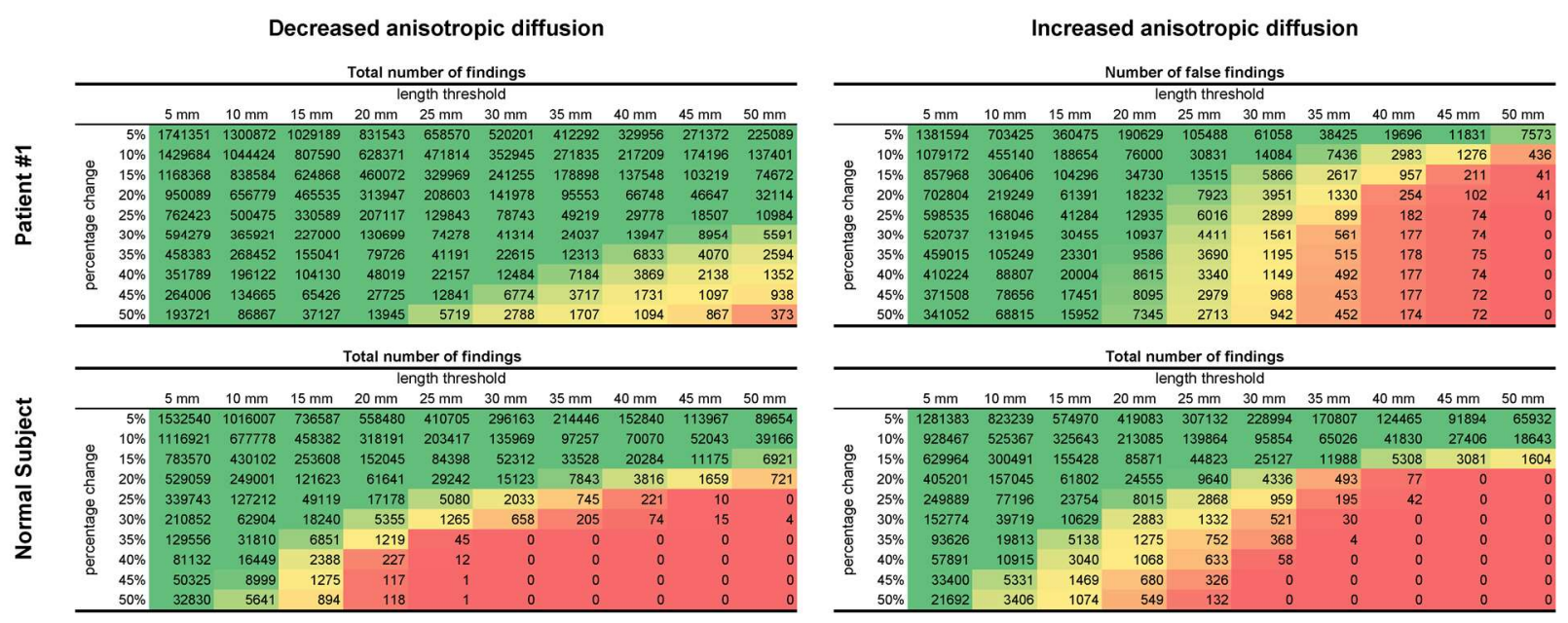

d

False discovery rate (FDR)

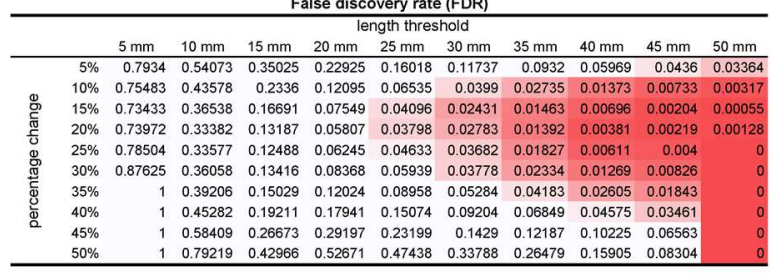




\section{Fig. 5}

701 Reliability assessment of differential tractography using the length threshold. (a) Differential

702 tractography is applied to a multiple sclerosis patient at different length thresholds. Only the tracks with

703 decreased anisotropy in the patient may contain true positive findings. A longer length threshold (e.g. >

$70440 \mathrm{~mm}$ ) can reduce false findings at the expense of sensitivity, whereas a shorter threshold may

705 introduce more false results. (b) Differential tractography is applied to a normal subject, and any findings

706 in the normal subject are false positives for neuronal injury. (c) The numbers of findings at different

707 length thresholds and change thresholds are listed in tables. The patient has substantially large

708 numbers of tracks with decreased anisotropy, suggesting a possible neurological injury. In comparison,

709 the normal subject has similar numbers of tracks with increased and decreased anisotropy. (d) False

710 discovery rate (FDR) of the findings in a patient can be calculated by using the patient's own numbers of

711 tracks with increased anisotropy as an estimation of the number of false findings. This allows for

712 adjusting the sensitivity and specificity of differential tractography and quantifying the reliability at

713 different length and change thresholds. 

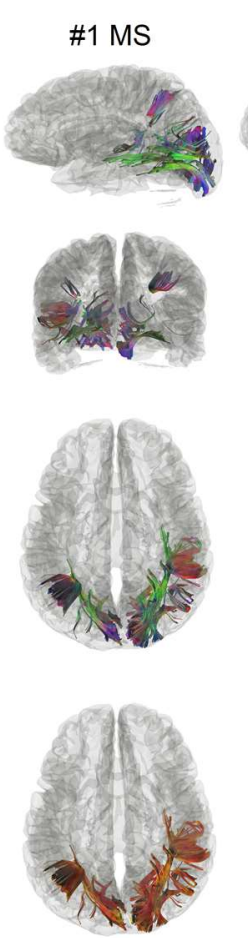
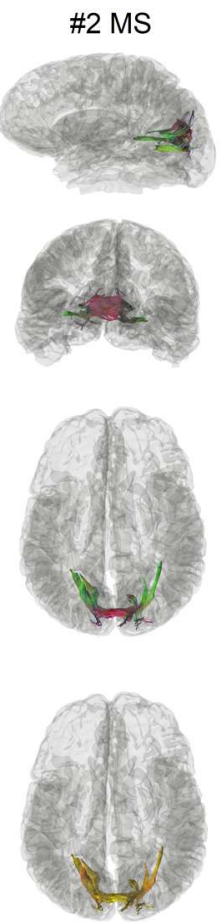
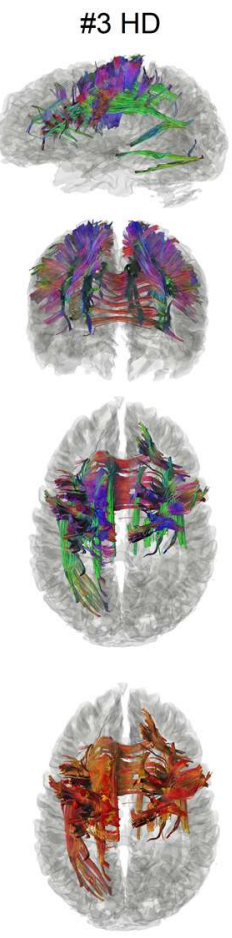
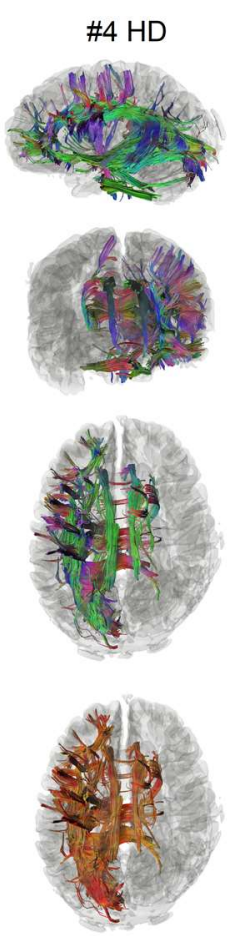
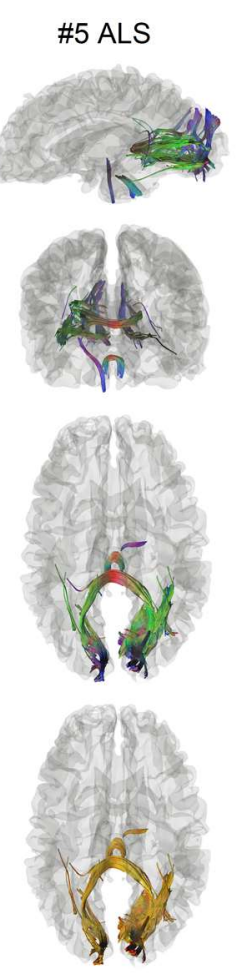
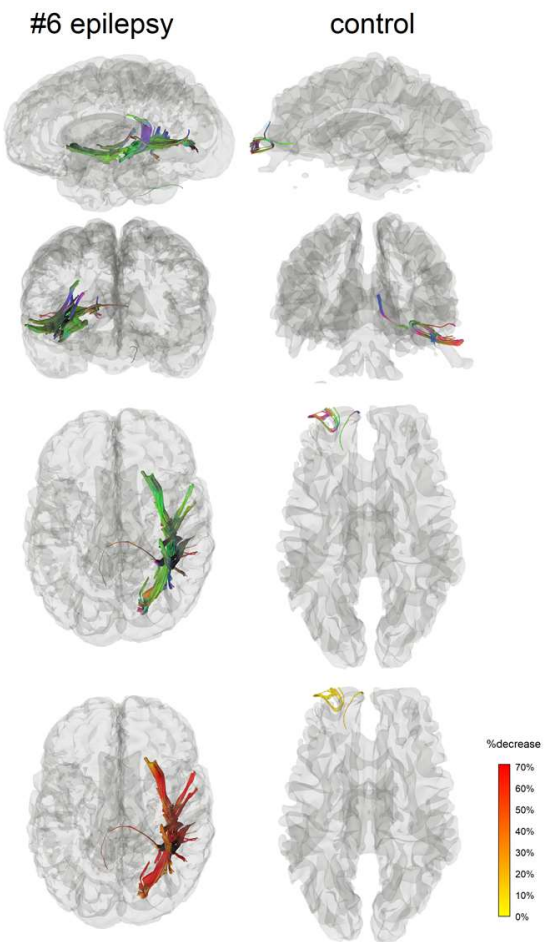

Fig. 6

717 Differential tractograms of patients with different neurological disorders in comparison with a normal

718 subject. The results were generated automatically without expert selection. The differential tractograms

719 of the two MS patients match well with their clinical presentation of optic neuritis. Patient \#1 has a much

720 severer drop in visual acuity, which can be quantitatively reflected by her larger the volume of the

721 findings and a greater percentage decrease of the anisotropy along the affected pathways. The

722 differential tractograms of the two Huntington diseases show extensive affected striatal pathways.

723 Patient \#4 had more asymmetric dystonia, matching the asymmetry presentation of the differential

724 tractography. The ALS patient had lower motor neuron presentation of left-hand weakness, matching 725 the finding of right lower corticospinal pathways in differential tractography. The epileptic patients 726 received right anterior temporal lobectomy, matching the findings in the differential tractogram that 727 shows the affected pathways around the surgical location. The false findings in the normal subject can 
bioRxiv preprint doi: https://doi.org/10.1101/576025; this version posted July 16, 2019. The copyright holder for this preprint (which was not certified by peer review) is the author/funder, who has granted bioRxiv a license to display the preprint in perpetuity. It is made available under aCC-BY 4.0 International license.

728 be differentiated by their less significant decrease of anisotropy and their location at the anterior frontal 729 region, which is known to be more susceptible to phase distortion artifact. 

aCC-BY 4.0 International license.

\section{a}

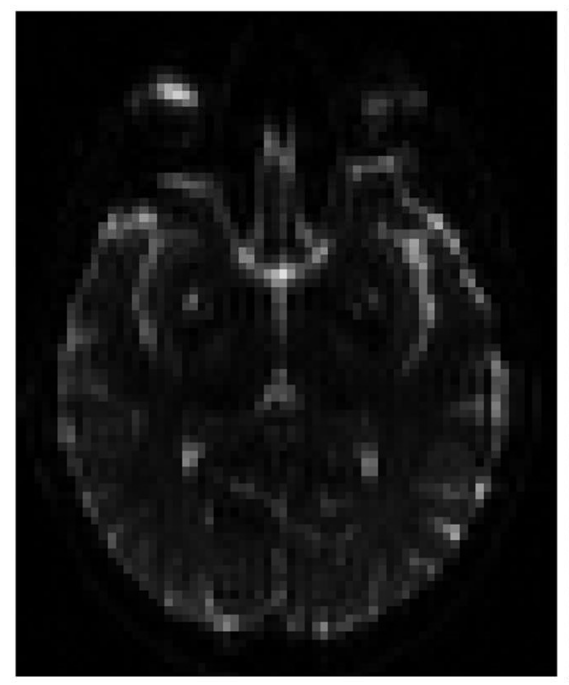

$\mathrm{b}=0 \mathrm{~s} / \mathrm{mm}^{2}$

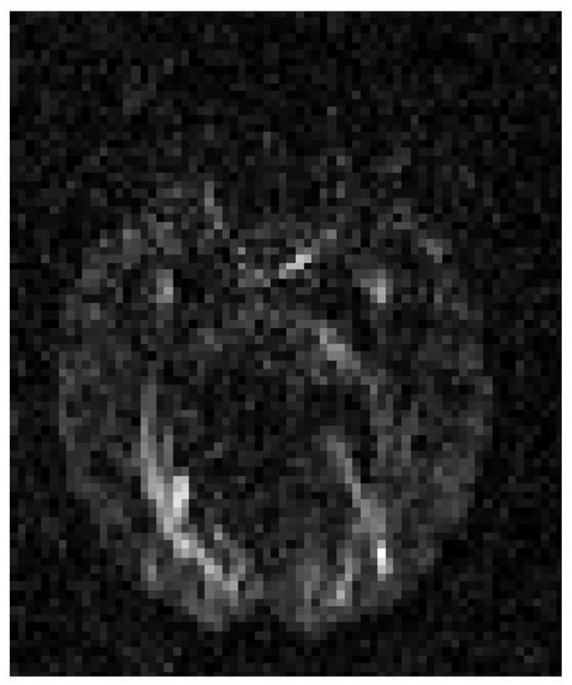

$\mathrm{b}=3000 \mathrm{~s} / \mathrm{mm}^{2}$

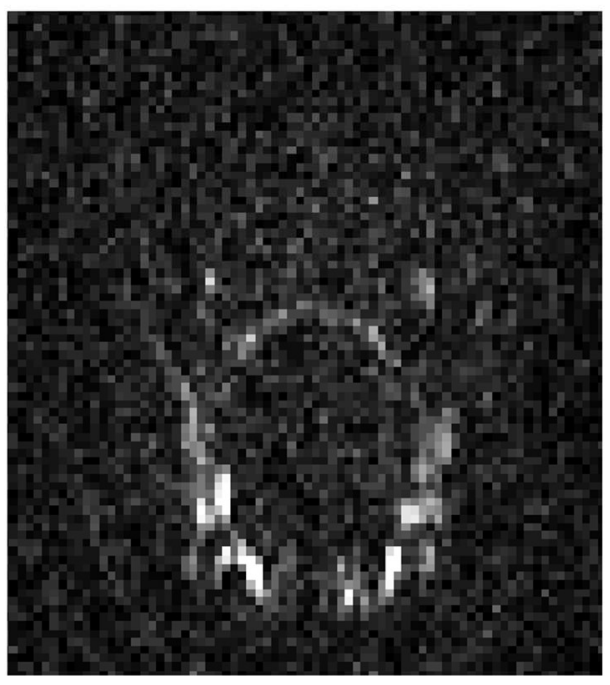

$\mathrm{b}=7000 \mathrm{~s} / \mathrm{mm}^{2}$

\section{b}
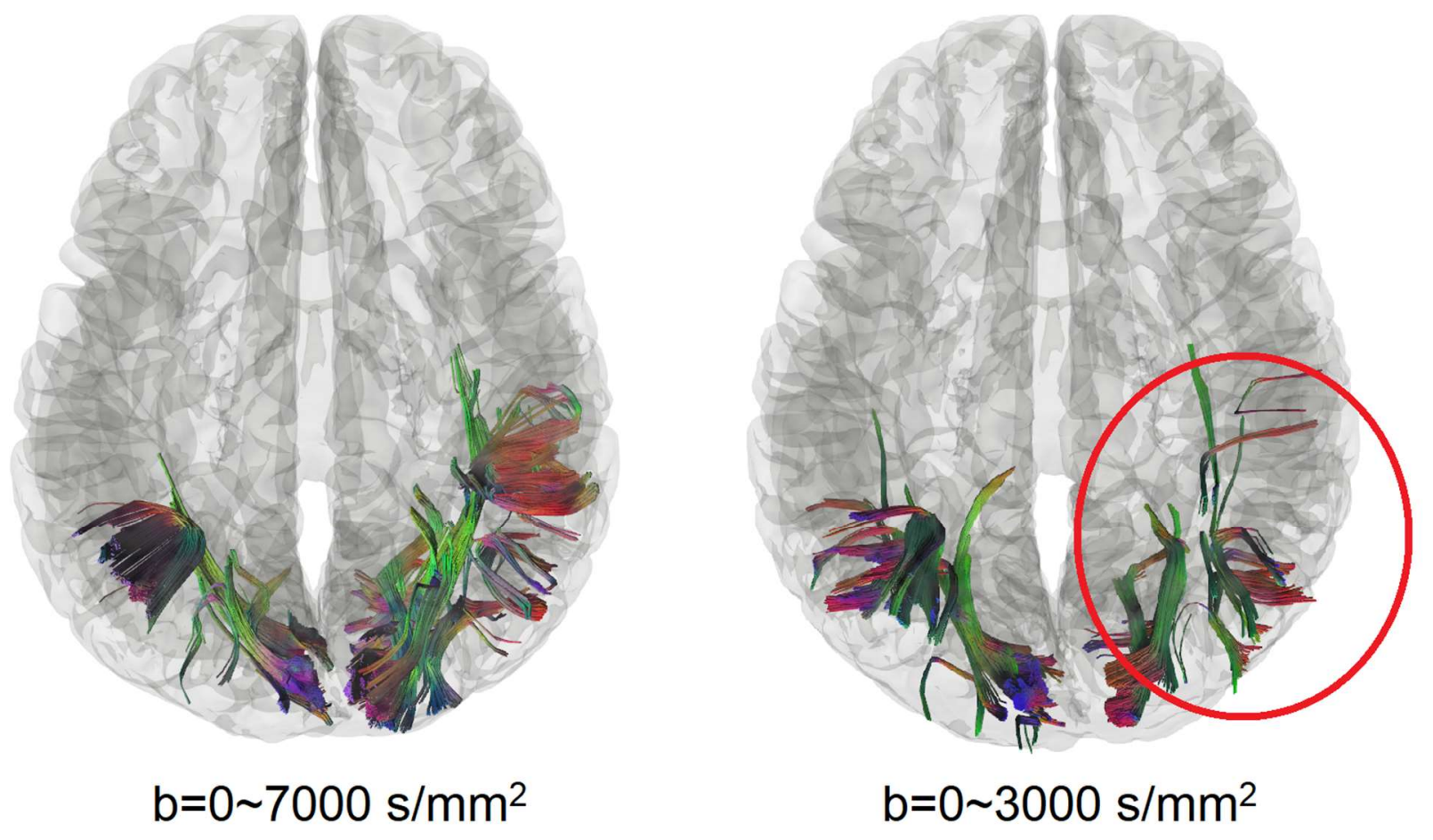

Fig. 7 
733 Performance differences in differential tractography due to different b-values. (a) Diffusion-weighted

734 images of a patient at b-values of 0,3000 , and $7000 \mathrm{~s} / \mathrm{mm}^{2}$. Signals at high b-values are sensitive to 735 restricted diffusion but have a lower signal-to-noise ratio. Thus most clinical scans only acquire b-value 736 lower than $3000 \mathrm{~s} / \mathrm{mm}^{2}$. (b) Differential tractography using reduced b-values between 0 and $3,000 \mathrm{~s} / \mathrm{mm}^{2}$ 737 shows $54 \%$ fewer findings than those from the full dataset using b-values between 0 and $7,000 \mathrm{~s} / \mathrm{mm}^{2}$. 738 Although the reduced b-value dataset also shows a grossly similar result, its FDR is substantially higher $739(\mathrm{FDR}=0.32)$ and thus not as reliable as the full dataset that includes high b-value data. This indicates the 740 important role of high b-value acquisition in detecting early neuronal injury. 
Table 1: Patient Demographics and Major Symptoms

\begin{tabular}{|c|c|c|c|c|c|}
\hline & diagnosis & age & sex & MRI scans & Major symptoms \\
\hline \#1 & $\begin{array}{l}\text { multiple } \\
\text { sclerosis }\end{array}$ & 44 & $\mathrm{~F}$ & $\begin{array}{l}\text { onset of symptom and } \\
\text { 6-month follow-up }\end{array}$ & $\begin{array}{l}\text { acute onset of left ocular pain, pain } \\
\text { with eye movements, blurring vision } \\
\text { of the left eye (20/400), loss of } \\
\text { visual field in all quadrants. }\end{array}$ \\
\hline \#2 & $\begin{array}{l}\text { multiple } \\
\text { sclerosis }\end{array}$ & 24 & $\mathrm{~F}$ & $\begin{array}{l}\text { onset of symptom and } \\
6 \text {-month follow-up }\end{array}$ & $\begin{array}{l}\text { acute onset of left ocular pain, pain } \\
\text { with eye movements, blurring vision } \\
\text { of the right eye (20/125), superior } \\
\text { altitudinal visual field defect. }\end{array}$ \\
\hline \#3 & $\begin{array}{l}\text { Huntington } \\
\text { disease }\end{array}$ & 60 & $M$ & $\begin{array}{l}\text { two scans at } 5 \text { months } \\
\text { apart during the manifest } \\
\text { stage }\end{array}$ & $\begin{array}{l}\text { body bradykinesia on both sides } \\
\text { UHDRS motor: } 45 \rightarrow 49 \text { (worsening) }\end{array}$ \\
\hline$\# 4$ & $\begin{array}{l}\text { Huntington } \\
\text { disease }\end{array}$ & 55 & $\mathrm{~F}$ & $\begin{array}{l}\text { two scans at } 5 \text { months } \\
\text { apart during the manifest } \\
\text { stage }\end{array}$ & $\begin{array}{l}\text { dystonia of left upper extremity, } \\
\text { dragging right foot with ambulation, } \\
\text { dystonia of the right leg } \\
\text { UHDRS motor: } 53 \rightarrow 64 \text { (worsening) }\end{array}$ \\
\hline \#5 & ALS & 48 & $M$ & $\begin{array}{l}\text { baseline scan acquired } 30 \\
\text { months after onset } \\
\text { follow-up scan acquired } \\
\text { one year after. }\end{array}$ & $\begin{array}{l}\text { left-hand weakness with } \\
\text { fasciculations } \\
\text { ALSFRS-R: } 45 \rightarrow 32 \text { (worsening) }\end{array}$ \\
\hline \#6 & epilepsy & 51 & $M$ & $\begin{array}{l}\text { before anterior temporal } \\
\text { lobectomy and one-year } \\
\text { follow-up after surgery }\end{array}$ & \\
\hline
\end{tabular}

ALS: amyotrophic lateral sclerosis, UHDRS: Unified Huntington's Disease Rating Scale, ALSFRS-R:

Amyotrophic Lateral Sclerosis Functional Rating Scale-Revised 


\section{Table 2: False Discovery Rate of Differential Tractography Findings}

\begin{tabular}{lcccrrr}
\hline & diagnosis & $\begin{array}{c}\text { change } \\
\text { threshold }\end{array}$ & $\begin{array}{c}\text { length } \\
\text { threshold }\end{array}$ & $\begin{array}{c}\text { number of } \\
\text { findings }\end{array}$ & $\begin{array}{c}\text { false-positive } \\
\text { findings }\end{array}$ & FDR \\
\hline \#1 & multiple sclerosis & $30 \%$ & $40 \mathrm{~mm}$ & 13,947 & 177 & $\leq 0.0126$ \\
$\# 2$ & multiple sclerosis & $30 \%$ & $40 \mathrm{~mm}$ & 2,799 & 0 & $<0.0001$ \\
$\# 3$ & Huntington disease & $30 \%$ & $40 \mathrm{~mm}$ & 85,243 & 3,571 & $\leq 0.0419$ \\
$\# 4$ & Huntington disease & $30 \%$ & $40 \mathrm{~mm}$ & 64,272 & 0 & $<0.0001$ \\
$\# 5$ & ALS & $15 \% \dagger$ & $40 \mathrm{~mm}$ & 12,222 & 2,548 & $\leq 0.2085$ \\
$\# 6$ & epilepsy post-op & $30 \%$ & $40 \mathrm{~mm}$ & 15,959 & 0 & $<0.0001$ \\
\hline & normal control & $30 \%$ & $40 \mathrm{~mm}$ & 74 & & \\
\hline
\end{tabular}

* estimated by the number of tracks with increased anisotropic diffusion $\uparrow 30 \%$ yields no findings 
Table 3: Differential Tractography Using Reduced Datasets $\left(b=0 \sim 3000 \mathrm{~s} / \mathrm{mm}^{2}\right)$

\begin{tabular}{lcrrr}
\hline & diagnosis & $\begin{array}{r}\text { number of findings and } \\
\text { its change(\%)† }\end{array}$ & $\begin{array}{r}\text { false-positive } \\
\text { findings* }\end{array}$ & FDR \\
\hline \#1 & multiple sclerosis & $6,454(-53.72 \%)$ & 2,071 & $\leq 0.3208$ \\
$\# 2$ & multiple sclerosis & $507(-81.89 \%)$ & 0 & $<0.0001$ \\
$\# 3$ & Huntington disease & $2,437(-97.14 \%)$ & 865 & $\leq 0.3549$ \\
$\# 4$ & Huntington disease & $39,903(-37.92 \%)$ & 0 & $<0.0001$ \\
$\# 5$ & ALS & $2,211(-81.91 \%)$ & 473 & $\leq 0.2139$ \\
$\# 6$ & epilepsy post-op & $7464(-53.23 \%)$ & 360 & $\leq 0.0482$ \\
\hline & normal control & $636(+759.46 \%)$ & & \\
\hline
\end{tabular}

*estimated by the number of tracks with increased anisotropic diffusion

tthe change is calculated by $(a-b) / b$, $a$ : current value $b$ : previous value 


\section{Supplementary Materials}

\section{Patient's medical history during the scan interval}

749 MS patients

750 Patient \#1 was a 44-year-old female with acute onset of left ocular pain and pain with eye movements.

751 She experienced a gradual decline in her visual acuity, and initial visual examination showed normal 752 acuity in the right eye and decreased acuity in the left eye (20/70), which worsen to 20/400 in one week.

753 She had a dense superior visual field defect in both superior quadrants that continued to worsen over a

754 few days with gradual loss of the inferior visual fields. She was treated with IV steroids, and after the 755 treatment, the ocular pain, pain with eye movement, and the visual field were improved. After 6 months 756 of her initial presentation, her visual acuity returned to $20 / 20$ both eyes and the visual fields were normal

757 Patient \#2 was a 23-year-old female with the blurring of vision of the right eye, associated with ocular

758 pain and pain with extraocular movements. Initial evaluation by ophthalmology noted visual acuity of $75920 / 40$ with enlargement of the blind spot on the Humphrey visual field. Within a week her symptoms 760 progressed to a visual acuity of $20 / 125$, decrease in the color perception. and a superior altitudinal visual 761 field defect. She was treated with intravenous methylprednisolone according to the optic neuritis 762 treatment trial. Her symptoms recovered within 10 days to visual acuity of $20 / 25$ and normal color 763 saturation.

$764 \quad H D$ patients 
765 Patient \#3 was a 60 -year-old male. The age of onset was 56, and the CAG repeats were 42. During the 766 scan interval, the UHDRS motor total was increased from 45 to 49 , with most prominent symptoms of 767 body bradykinesia on both sides.

768 Patient \#4 was a 55-year-old female. The age of onset was 48 , and the CAG repeats were 43 . During 769 the scan interval, the UHDRS motor total was increased from 53 to 64, with symptoms of dystonic 770 contractures of the left upper extremity, dragging right foot with ambulation, and dystonia in the legs 771 more obvious with gait (mostly on the right side), moderate bradykinesia with the finger taps (mostly on 772 the left side).

773 ALS patient

774 Patient \#5 was a 48-year-old male ALS patient (laboratory-supported probable ALS diagnosis) with 775 upper extremity limb onset and predominant lower motor neuron involvement. His symptoms started 776 about 30 months before the baseline scan with widespread fasciculations and left-hand weakness. 777 There were subtle upper motor neuron signs in cervical segments. He developed lower extremity 778 weakness 2 years after onset but never developed lower extremity upper motor neuron signs at the time 779 of death that occurred 5 years after the onset of symptoms. He had no family history of neurological 780 disease. The patient's ALSFRS-R score decreased from 45 to 32 during the MRI scan interval (1 year 781 apart).

Epilepsy patient

783 Patient \#6 was a 51-year-old male with medically intractable epilepsy since the age of 17 . His seizure 784 frequency was weekly, and intracranial monitoring demonstrated that his seizure onset zone included 785 the right mesial and neocortical temporal lobe. He underwent a standard right anterior temporal lobectomy. MRI scans were obtained before the surgery and one year after the surgery. 
bioRxiv preprint doi: https://doi.org/10.1101/576025; this version posted July 16,2019 . The copyright holder for this preprint (which was not certified by peer review) is the author/funder, who has granted bioRxiv a license to display the preprint in perpetuity. It is made available under aCC-BY 4.0 International license. 